\title{
Multiscale formulation for coupled flow-heat equations arising from single-phase flow in fractured geothermal reservoirs
}

\author{
Timothy Praditia ${ }^{1,2,3} \cdot$ Rainer Helmig ${ }^{2} \cdot$ Hadi Hajibeygi ${ }^{1}$ (i)
}

Received: 18 November 2017 / Accepted: 5 June 2018 / Published online: 20 June 2018

(C) The Author(s) 2018

\begin{abstract}
Efficient heat exploitation strategies from geothermal systems demand for accurate and efficient simulation of coupled flow-heat equations on large-scale heterogeneous fractured formations. While the accuracy depends on honouring highresolution discrete fractures and rock heterogeneities, specially avoiding excessive upscaled quantities, the efficiency can be maintained if scalable model-reduction computational frameworks are developed. Addressing both aspects, this work presents a multiscale formulation for geothermal reservoirs. To this end, the nonlinear time-dependent (transient) multiscale coarse-scale system is obtained, for both pressure and temperature unknowns, based on elliptic locally solved basis functions. These basis functions account for fine-scale heterogeneity and discrete fractures, leading to accurate and efficient simulation strategies. The flow-heat coupling is treated in a sequential implicit loop, where in each stage, the multiscale stage is complemented by an ILU(0) smoother stage to guarantee convergence to any desired accuracy. Numerical results are presented in 2D to systematically analyze the multiscale approximate solutions compared with the fine scale ones for many challenging cases, including the outcrop-based geological fractured field. These results show that the developed multiscale formulation casts a promising framework for the real-field enhanced geothermal formations.
\end{abstract}

Keywords Geothermal reservoir simulation · Fractured porous media $\cdot$ Coupled mass-heat transfer $\cdot$ Multiscale finite volume method

\section{Introduction}

Production stimulation in enhanced geothermal systems depends heavily on activation of fractures, in order to

Timothy Praditia

timothy.praditia@iws.uni-stuttgart.de

Rainer Helmig

Rainer.Helmig@iws.uni-stuttgart.de

Hadi Hajibeygi

H.Hajibeygi@tudelft.nl

1 Department of Geosciences and Engineering, Faculty of Civil Engineering and Geosciences, TU Delft, Stevinweg 1, 2628 CN Delft, The Netherlands

2 Department of Hydromechanics and Modelling of Hydrosystems, Institute for Modelling Hydraulic and Environmental Systems, Universität Stuttgart, Pfaffenwaldring 61, 70569 Stuttgart, Germany

3 Present address: Department of Stochastic Simulation and Safety Research for Hydrosystems, Institute for Modelling Hydraulic and Environmental Systems, Universität Stuttgart, Pfaffenwaldring 5a, 70569 Stuttgart, Germany increase the effective formation conductivity. These formations are naturally developed over large $(\mathrm{km})$ length scales, while the heterogeneity of the damaged matrix needs to be resolved at fine (e.g., $\mathrm{cm}$ ) scales. Fractures naturally add to the complexity of the mathematical formulations by introducing significant contrasts in the conductivity and geometry [1-4]. Their important role in the flow and transport of mass and energy can be properly investigated only if they are explicitly represented in the computational domain [5-12].

The embedded discrete fracture model (EDFM) [13-15] has been developed to resolve several geometrical challenges due to explicit treatment of the fractures. EDFM has been extended to complex scenarios in multiphase iso-thermal reservoir simulation [16-18], and, importantly, to geothermal systems [19]. Recently, a consistent projection-based EDFM (pEDFM) for flow has been proposed to account for all types of fracture conductivities (from flow barriers to high conductive channels) [20].

The size of final fine-scale systems describing single phase flow in fractured geothermal reservoirs, even after EDFM modeling approach, is beyond the scope of state-of-the-art 
commercial simulators. Upscaling these highly heterogeneous discrete quantities - in order to reduce the computational costs - would lead to inaccurate simulations, with no error control ability to the reference system. Therefore, new modeling and simulation techniques are more than ever on demand.

Multiscale finite volume methods have been developed for resolving this computational challenge by constructing coarse-scale systems based on local basis functions [21, 22]. They are mainly developed for flow equations with complex fluid physics [23]. Together with their recent developments for fractured media [10, 15, 24-27], they form a promising approach for real-field applications. For geothermal applications, however, the coupled flowheat equations need to be considered, which leads to additional complexities both in linear (size of the discrete system) and nonlinear (temperature-dependent coefficients and nonlinear coupling terms) aspects [28].

In this work, we propose a multiscale formulation for coupled flow-heat equations in fractured porous media, where not only the flow but also the heat equation are mapped to the coarse-scale system by using local basis functions. We investigate two different matrix-fracture coupling procedure for heat and flow basis functions, namely totally independent and semi-dependent. These two approaches differ from each other by the amount of matrixfracture coupling and number of matrix basis functions in the local system construction. The nonlinear coupling between flow and heat is treated with a sequential implicit approach.

Several challenging test cases are considered where the fractures play major role in transport of the cold water into the reservoir, and thus enhancing the production of heat. The large temperature gradients (due to slow to fast flow field) adds to the complexity of the simulations, which form a good basis to investigate the accuracy of the developed multiscale method. For all the investigated test cases, including the outcrop-based characterized formation, the basis function formulation for both the flow and heat equations are shown to be able to approximate the reference fine-scale solutions very well. Specially, the very first approximate multiscale solutions (with no iterations) are compared with the fine-scale solutions after the first Newton update, with very close agreement. Note that, even though a single-phase flow is considered here, the conservative velocity field of the Multiscale Finite Volume (MSFV) method is crucial for accurate transport of enthalpy which appears in the heat balance equation. The proposed multiscale finite volume method therefore casts a promising approach for field-scale geothermal studies.

Note that this work focuses on developing an accurate method to approximate the fine scale (fully resolved) solution. While the efficiency improvement is also part of the goal of the multiscale method, it is not extensively studied in this work and would be subject to future studies and development. However, the efficiency of the multiscale method for pressure solver in fractured reservoirs has been studied, and interested readers are referred to [24].

This paper is organized as follows. First, the mass and energy conservation equation of single-phase water system in fractured porous media are presented, together with the coupling strategy used to calculate pressure and temperature. Then, the MSFV method is introduced, and its application for pressure and temperature calculation is explained. After that, the numerical results are presented and discussed, including the simulation results of a realfield fracture geometry taken from outcrop data. Finally, the conclusions are presented.

\section{Governing equations}

\subsection{Mass conservation equation}

Mass conservation for single-phase flow in fractured porous media with embedded discrete fracture modelling (EDFM) approach reads

$$
\left[\frac{\partial}{\partial t}(\phi \rho)-\nabla \cdot(\rho \lambda \cdot \nabla p)\right]^{m}=[\rho q]^{m w}+[\rho q]^{m f}
$$

for matrix on $\Omega^{m} \subset R^{n}$, and

$$
\left[\frac{\partial}{\partial t}(\phi \rho)-\nabla \cdot(\rho \lambda \cdot \nabla p)\right]^{f}=[\rho q]^{f w}+[\rho q]^{f m}
$$

on $\Omega^{f} \subset R^{n-1}$ for fractures. In this work, $n=2$. But these general formulations are also valid for three-dimensional domain (i.e., $n=3$ ). Note that if gravitational effects are considered, one has to replace the pressure $p$ with the potential $(p-\rho g z)$, where $\rho, g$, and $z$ are, respectively, density, gravitational acceleration, and the coordinate along the gravity (pointing downward). Moreover, the superscripts $m, f$, and $w$ indicate, respectively, the matrix, fracture, and well (source) terms. Moreover, $\phi$ stands for the porosity, $\lambda$ the mobility, and $q$ the flow rate. Note that the equation for fractures is defined in a lower dimensional space than for the matrix.

The mobility is defined as $\lambda=k / \mu$, where $k$ is the absolute permeability and $\mu$ is the water viscosity. While the matrix permeability is characterized from geological inputs (possibly after proper upscaling), the permeability of the fractures can be defined (under fully developed flow assumption between parallel plates of distance (aperture) $a$ ) as $k^{f}=a^{2} / 12$.

The well flow rates read

$q^{m w}=\frac{P I \lambda\left(p^{w}-p^{m}\right)}{V} \equiv \beta^{m}\left(p^{w}-p^{m}\right)$, 
for matrix and

$q^{f w}=\frac{P I \lambda\left(p^{w}-p^{f}\right)}{A} \equiv \beta^{f}\left(p^{w}-p^{f}\right)$,

for fractures, where $P I$ is the well productivity index [29], and $\beta$ is the normalized well productivity index, with $\beta^{m}$ normalized with the matrix control volume $V$ (i.e., $\beta^{m}=$ $(P I \lambda) / V)$ and $\beta^{f}$ normalized with the fracture area $A$ (i.e., $\left.\beta^{f}=(P I \lambda) / A\right)$.

The discrete flux exchange between matrix and fractures, i.e., $q^{m f}$ and $q^{f m}$, are modeled using EDFM approach as

$q^{m f}=\frac{C I \lambda^{f-m}\left(p^{f}-p^{m}\right)}{V} \equiv \eta^{m}\left(p^{f}-p^{m}\right)$,

and

$q^{f m}=\frac{C I \lambda^{f-m}\left(p^{m}-p^{f}\right)}{A} \equiv \eta^{f}\left(p^{m}-p^{f}\right)$.

Here, $C I$ is the connectivity index between matrix and fracture, $\lambda^{f-m}$ is the effective mobility at matrix-fracture interface, and $\eta$ is the normalized connectivity index, with $\eta^{m}$ normalized with the matrix control volume $V$ (i.e., $\eta^{m}=$ $C I \lambda^{f-m} / V$ and $\eta^{f}$ normalized with fracture area $A$ (i.e. $\eta^{f}=C I \lambda^{f-m} / A$, [24]. The discrete connectivity index $C I$ allows for the representation of the discrete fracture element $i$ overlapping with the matrix element $j$, i.e.,

$C I_{i-j}=\frac{A_{i-j}}{\langle d\rangle_{i-j}}$,

where $A_{i-j}$ the surface area of fracture element $i$ inside the element $j$, and $\langle d\rangle_{i-j}$ is the average normal distance between the two elements [15].

\subsection{Energy conservation equation}

In this study, local thermal equilibrium between fluid and solid is assumed [30-32], i.e., the rock and the fluid have the same temperature at any given location. Under this assumption, the single-phase energy conservation equation reads

$\left[\frac{\partial}{\partial t}\left(\phi \rho U+(1-\phi) \rho_{r} C_{p r} T\right)+\nabla \cdot(u h)\right.$

$$
\left.-\nabla \cdot\left(\lambda_{c} \cdot \nabla T\right)\right]^{m}=\left[q_{c}+q_{H}\right]^{m f}+\left[q_{H}\right]^{m w}
$$

on $\Omega^{m} \subset R^{n}$ for matrix, and

$$
\begin{aligned}
& {\left[\frac{\partial}{\partial t}\left(\phi \rho U+(1-\phi) \rho_{r} C_{p r} T\right)+\nabla \cdot(u h)\right.} \\
& \left.\quad-\nabla \cdot\left(\lambda_{c} \cdot \nabla T\right)\right]^{f}=\left[q_{c}+q_{H}\right]^{f m}+\left[q_{H}\right]^{f w}
\end{aligned}
$$

on $\Omega^{f} \subset R^{n-1}$ for fractures. Here, $\rho_{r}$ and $C_{p r}$ are the density and the specific heat capacity of the rock, $U$ and $h$ are the water specific internal energy and specific enthalpy, respectively. Also, $T$ is the temperature and $\lambda_{c}$ is the average thermal conductivity, which is computed as $\lambda_{c}=\phi \lambda_{c w}+$ $(1-\phi) \lambda_{c r}$. The well source term, $q_{H}^{* w}$ is defined as

$q_{H}^{* w}=[\rho q h]^{* w}$,

with $q^{* w}$ defined in Eqs. 3 and 4. Finally, $u$ is the mass flow rate, which reads $u=-\rho \lambda \cdot \nabla p$ according to Darcy's law.

From Eqs. 8 and 9, the matrix-fracture heat coupling is divided into two parts: conduction and convection. The conduction coupling terms $q_{c}$ are defined analogous to the matrix-fracture mass (flow) transfer, i.e.,

$q_{c}^{m f}=\frac{C I \lambda_{c}^{f-m}\left(T^{f}-T^{m}\right)}{V} \equiv \eta_{c}^{m}\left(T^{f}-T^{m}\right)$,

and

$q_{c}^{f m}=\frac{C I \lambda_{c}^{f-m}\left(T^{m}-T^{f}\right)}{A} \equiv \eta_{c}^{f}\left(T^{m}-T^{f}\right)$,

where $\eta_{c}$ is the normalized conductive connectivity index; with $\eta_{c}^{m}$ normalized with the matrix control volume $V$ (i.e. $\eta_{c}^{m}=C I \lambda_{c}^{f-m} / V$ and $\eta_{c}^{f}$ normalized with fracture area $A$ (i.e. $\eta_{c}^{f}=C I \lambda_{c}^{f-m} / A$.

The convection coupling terms, $q_{H}$, are defined based on the exchange mass between the two media, i.e., $q^{m f}$ and $q^{f m}$, as

$q_{H}^{m f}=[\rho q h]^{m f}$,

and

$q_{H}^{f m}=[\rho q h]^{f m}$.

Note that the convection and conduction heat transfer are defined in a strictly conservative manner, i.e.,

$\int_{V} q_{c}^{m f} d V=-\int_{A} q_{c}^{f m} d A$,

and

$\int_{V} q_{H}^{m f} d V=-\int_{A} q_{H}^{f m} d A$

hold.

\subsection{Sequential implicit simulation strategy}

The fluid properties depend on both pressure and temperature (see Appendix A for detailed formulations). These properties result in non-linearity of each mass and heat transfer equation, as well as the co-dependency between the coupled equations.

A sequential implicit formulation is followed to treat the nonlinearly coupled mass and heat transfer equations for both fine-scale and multiscale simulations, where the pressure equation is first solved, then the total velocities are obtained and then the temperature solution in the fractured media is obtained. Notice that these equations are nonlinear functions of pressure and temperature and therefore are solved implicitly using the Newton-Raphson method. 
The linearized equation for the general unknown $x$ (i.e., $\boldsymbol{p}$ or $\boldsymbol{T}$ ) reads

$\boldsymbol{A}_{x}^{v} \cdot \boldsymbol{x}^{\nu+1}=\boldsymbol{f}_{x}^{v}$

or, in expanded form,

$$
\left[\begin{array}{lll}
\boldsymbol{A}_{x}^{m m} & \boldsymbol{A}_{x}^{m f} & \boldsymbol{A}_{x}^{m w} \\
\boldsymbol{A}_{x}^{f m} & \boldsymbol{A}_{x}^{f f} & \boldsymbol{A}_{x}^{f w} \\
\boldsymbol{A}_{x}^{w m} & \boldsymbol{A}_{x}^{w f} & \boldsymbol{A}_{x}^{w w}
\end{array}\right]^{v}\left[\begin{array}{l}
\boldsymbol{x}^{m} \\
\boldsymbol{x}^{f} \\
\boldsymbol{x}^{w}
\end{array}\right]^{v+1}=\left[\begin{array}{l}
\boldsymbol{f}_{x}^{m} \\
\boldsymbol{f}_{x}^{f} \\
\boldsymbol{f}_{x}^{w}
\end{array}\right]^{v} .
$$

The superscript $v$ indicates the iteration stage, $\boldsymbol{A}$ the system matrix, and the vector $\boldsymbol{f}_{x}^{v}$ stands for the right-hand-side terms. Note that for each unknown $\boldsymbol{x}$, matrix, fracture, and well terms are present and that the system matrix and righthand-side terms depend on both $\boldsymbol{p}$ and $\boldsymbol{T}$. As such, the complexity of the system is quite significant for real-field applications.

To account for the co-dependency between both conservation equations, an outer loop iteration between pressure and temperature solver (see procedure in Algorithm 1) is needed. The outer loop runs until convergence is reached based on $\left\|\boldsymbol{\delta} \boldsymbol{p}^{\boldsymbol{v}+\mathbf{1}}\right\|_{2}$ and $\left\|\boldsymbol{\delta} \boldsymbol{T}^{\boldsymbol{v}+\mathbf{1}}\right\|_{2}$.

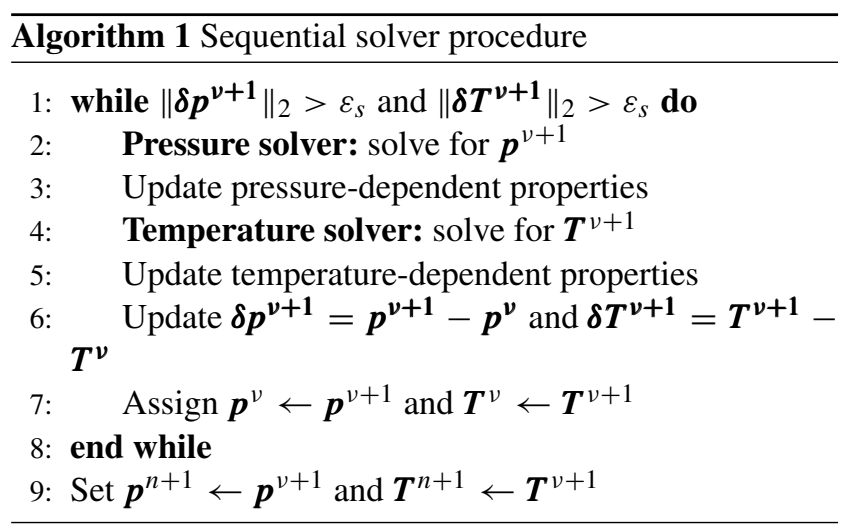

\section{MSFV method for nonlinear flow and heat equations}

MSFV method approximates the fine scale solution by superposing the coarse scale solution with the basis functions as the interpolator. The approximate solution is defined as

$$
\begin{aligned}
x^{*} \approx x^{*}= & \sum_{i=1}^{N_{c m}} \Phi_{i, x}^{* m} \breve{x}_{i}^{m}+\sum_{i=1}^{N_{f}} \sum_{j=1}^{N_{c f_{i}}} \Phi_{j, x}^{* f} \breve{x}_{j}^{f_{i}} \\
& +\sum_{k=1}^{N_{w}} \Phi_{k, x}^{* w} \breve{x}_{k}^{w},
\end{aligned}
$$

where $x$ is a generic term denoting the unknown (i.e., $p$ or $T), x^{\prime}$ the approximate fine scale solution, and $\breve{x}$ the coarse scale solution. The superscript $*$ indicates the domain on which the unknowns are defined (i.e., matrix or fracture), and $\Phi_{x}^{* \bullet}$ the local basis functions in domain $*$ coupled with domain • (i.e., matrix $(m)$, fracture $(f)$, or well $(w)$ ). Finally, $N_{f}$ is the number of fracture networks, $N_{c f_{i}}$ the number of primal coarse cell in fracture $i$, and $N_{w}$ the number of wells ( $N_{w, i n j}$ in temperature system, which is the number of injection wells).

\subsection{Multiscale grids}

In the MSFV methods, two types of coarse grids are constructed and imposed on the fine scale grids. The primal coarse cells are constructed as the coarse-scale control volumes, while the dual coarse grids are overlapping coarse grids bounded by the coarse nodes (vertices) on which the local basis functions are computed (see Fig. 1).

Figure 1 also illustrates the embedded fracture networks. Using EDFM benefits the multiscale implementation in that the coarsening strategy of the fracture elements is entirely independent of the matrix coarsening. Moreover, the fracture elements can be connected to any matrix cell, i.e., a vertex, edge, or an internal cell (based on dual-corsecell partition [33]).

\subsection{MSFV for the flow equation}

In development of an efficient multiscale method, the proper choice of basis function formulation is important. The important factors to consider for basis functions are their accuracy in representation of the underlying heterogeneity (accuracy), and their independency on the primary unknowns for adaptivity (efficiency). In this work, these aspects are being considered to formulate both pressure and temperature basis functions.

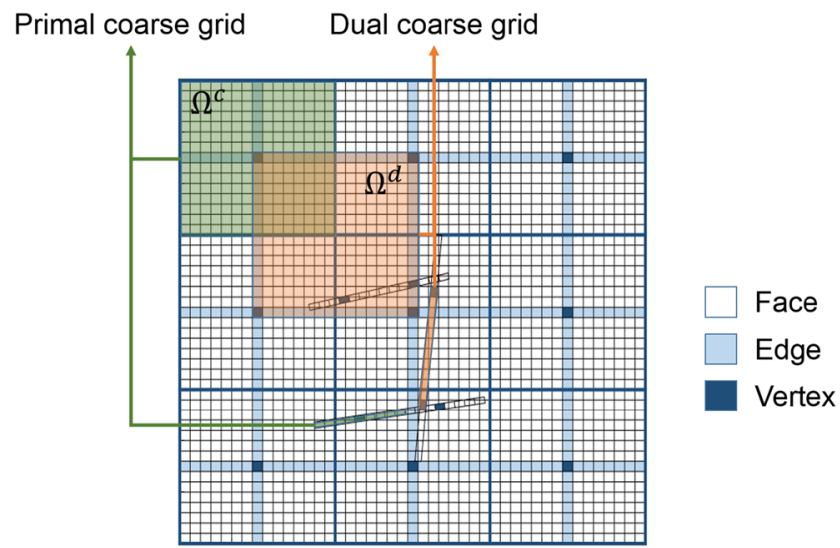

Fig. 1 Multiscale grids in 2D with $45 \times 45$ fine cells and coarsening ratio of 15 for a fractured domain. Note that fracture thickness (aperture) is exaggerated for presentation clarity 
The general formulation of the pressure basis function is written as

$$
\begin{array}{r}
-\nabla \cdot\left(\lambda^{*} \cdot \nabla \Phi_{p}^{* \bullet}\right)+\sum_{j \in \operatorname{conn}_{m f}^{*}} \eta_{j}^{*} \xi\left(\Phi_{p}^{* \bullet}\right) \\
+\sum_{j \in p e r f_{w}^{*}} \beta_{j}^{*}\left(\Phi_{p}^{* \bullet}-\Phi_{p}^{w \bullet}\right)=0,
\end{array}
$$

where $\Phi_{p}^{* \bullet} \in\left\{\Phi_{p}^{m m}, \Phi_{p}^{m f}, \Phi_{p}^{m w}, \Phi_{p}^{f m}, \Phi_{p}^{f f}, \Phi_{p}^{f w}\right\}$. The function $\xi\left(\Phi_{p}^{* \bullet}\right)$ is different for each coupling strategy.

Equation 20 is formulated based on an equivalent incompressible system equation. This formulation is proven [34] to be the most efficient strategy (based on CPU measurements) because it eliminates the need to frequently update the local basis function, while the fully compressible coarse-scale system takes care of the global compressibility (and time-dependent) effects.

The pressure basis function formulated using Eq. 20 needs to be calculated at the beginning of the simulation, and updated only if the water mobility $\lambda$ changes above a prescribed tolerance (i.e., due to the change of the water viscosity). Moreover, with regard to the matrixfracture coupling, we consider two different coupling strategies for the basis function calculation, namely the totally independent (fully decoupled) and semi-dependent (coupled) strategy. More precisely, the totally independent (decoupled) strategy formulates basis function without any coupling between matrix and fractures, while the semidependent strategy is formulated with partial coupling between matrix and fractures [24]. In the semi-dependent strategy, the matrix basis function is coupled with the fractures, but fracture basis function is decoupled from the matrix. This way, the semi-dependent approach enriches the matrix basis functions with the number of fracture course cells inside a dual-coarse domain.

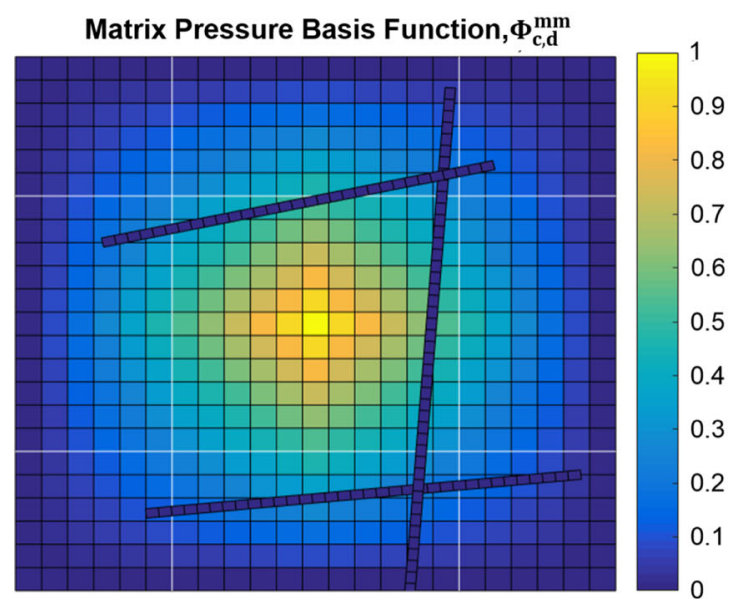

(a)

\subsubsection{Totally independent approach for basis functions}

In the totally independent coupling strategy, all basis functions are calculated independent of interactions with other domains, i.e.,

$\xi\left(\Phi_{p}^{* \bullet}\right)=0 \forall \Phi_{p}^{* \bullet} \in\left\{\Phi_{p}^{m m}, \Phi_{p}^{m f}, \Phi_{p}^{m w}, \Phi_{p}^{f m}, \Phi_{p}^{f f}, \Phi_{p}^{f w}\right\}$.

An example of the pressure basis function calculated using this strategy is shown in Fig. 2. In Fig. 2a, it is shown that the matrix basis function is not affected by fracture existence, as well as fracture basis function not affected by matrix basis function in Fig. 2b. The basis function forms a partition of unity, meaning that the sum of all the basis function is equal to 1 .

\subsubsection{Semi-dependent approach for basis functions}

In the semi-dependent coupling strategy, fracture basis function $\Phi_{p}^{f f}$ is first calculated, decoupled with the matrix basis function, using

$\xi\left(\Phi_{p}^{f f}\right)=0$.

These values are then used as Dirichlet boundary condition to calculate $\Phi^{m f}$ and setting

$\xi\left(\Phi_{p}^{m f}\right)=\Phi_{p}^{m f}-\Phi_{p}^{f f}$

to account for the connectivity of matrix basis function with the fracture domain. An example is shown in Fig. 3a, where $\Phi_{p}^{f f}$ is plotted in the fractures and $\Phi_{p}^{m f}$ is plotted in the matrix with the coupling effect clearly observed.

The matrix basis function $\Phi_{p}^{m m}$ is calculated by setting

$\xi\left(\Phi_{p}^{m m}\right)=\Phi_{p}^{m m}$,

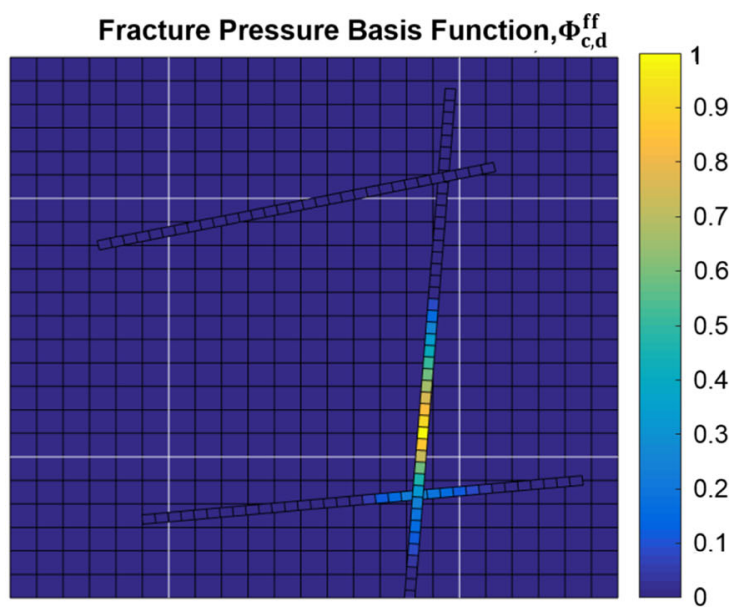

(b)

Fig. 2 Matrix basis function $\Phi_{p}^{m m}$ (a) and fracture basis function $\Phi_{p}^{f f}$ (b) calculated using the totally independent coupling strategy 


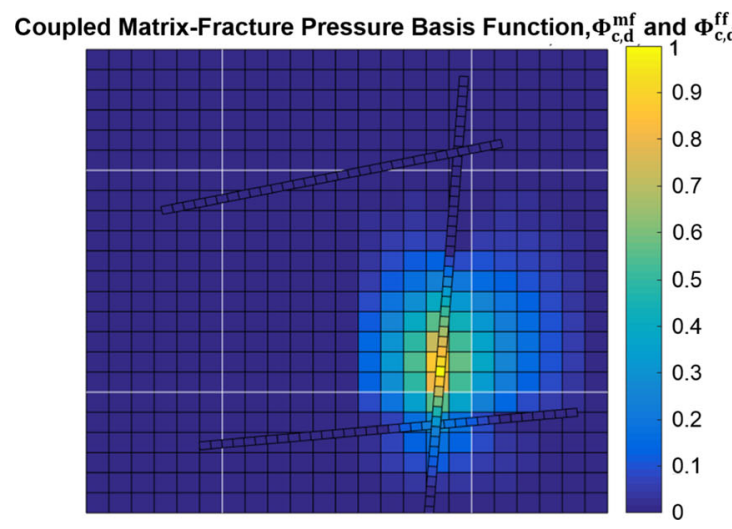

(a)

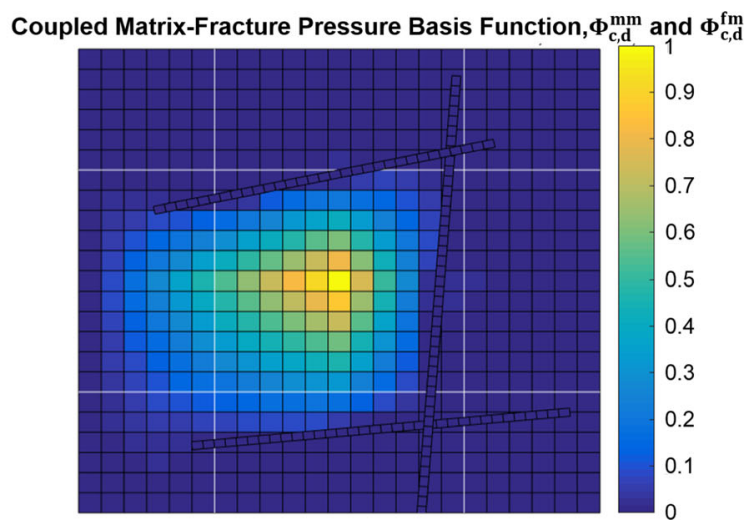

(b)

Fig. 3 a $\Phi_{p}^{m f}$ and $\Phi_{p}^{f f}$ and b) $\Phi_{p}^{m m}$ and $\Phi_{p}^{f m}$ calculated using semi-dependent coupling strategy

therefore also accounting for fracture existence. An example is shown in Fig. $3 b$ where the fracture basis function $\Phi_{p}^{f m}$ is set to 0 , and the matrix basis function $\Phi_{p}^{m m}$ observing the effect of the fracture existence, as though the fractures act as flow barriers. This strategy also results in partition of unity.

\subsubsection{Fine scale flux reconstruction}

In the pressure MSFV method, one of the most important step is the fine-scale conservative flux reconstruction. In MSFV, the mass fluxes are conservative only at coarse scale. Therefore, the fine scale fluxes need to be obtained via additional reconstruction step [35]. This is especially important in multiphase flows, to accurately predict the saturation front since the fractional flow is sensitive to the flux. In geothermal simulations, conservative mass flux is also needed in the convection part of the energy balance calculation due to its velocity dependency. Therefore, it is worth revisiting the fine scale flux reconstruction in this subsection.

The mass flow rate formulation

$\boldsymbol{u}^{\prime}=-\rho \lambda \cdot \nabla p^{\prime}$

is valid at the primal coarse cell boundaries $\partial \Omega_{c}$. The conservative fine-scale flux can be reconstructed after solving

$\left[\frac{\partial}{\partial t}(\phi \rho)-\nabla \cdot\left(\rho \lambda \nabla p_{c}^{\prime \prime}\right)\right]^{*}=[\rho q]^{* w}+[\rho q]^{* \bullet}$

locally on primal-coarse cells $\Omega_{c}$, subject to the boundary condition

$\left(\rho \lambda \nabla p_{c}^{\prime \prime}\right) \cdot \overline{\boldsymbol{n}}_{c}=\left(\rho \lambda \nabla p^{\prime}\right) \cdot \overline{\boldsymbol{n}}_{c}$

at $\partial \Omega_{c}$. Here, $\overline{\boldsymbol{n}}_{c}$ is the normal vector pointing out of the primal coarse cell boundaries, meaning that the fluxes at the coarse cell interfaces are used as Neumann boundary condition to calculate the reconstructed local pressure. The locally conservative mass flux is finally reconstructed as

$\boldsymbol{u}^{\prime \prime}= \begin{cases}-\rho \lambda \cdot \nabla p_{c}^{\prime \prime}, & \text { on } \Omega_{c} \\ -\rho \lambda \cdot \nabla p^{\prime}, & \text { at } \partial \Omega_{c} .\end{cases}$

\subsection{MSFV for the heat equation}

To exploit the efficiency of the temperature basis functions, they are formulated based on the conduction term within the whole energy balance equation. This allows for convenient implementation, as well as efficient algorithm (since basis functions are not required to be frequently updated).

The general formulation of the temperature basis function can be defined as

$-\nabla \cdot\left(\lambda_{c}^{*} \cdot \nabla \Phi_{T}^{* \bullet}\right)+\sum_{j \in \operatorname{conn}_{m f}^{*}} \eta_{c, j}^{*} \xi\left(\Phi_{T}^{* \bullet}\right)=0$

where $\Phi_{T}^{* \bullet} \in\left\{\Phi_{T}^{m m}, \Phi_{T}^{m f}, \Phi_{T}^{m w}, \Phi_{T}^{f m}, \Phi_{T}^{f f}, \Phi_{T}^{f w}\right\}$. The function $\xi\left(\Phi_{T}^{* \bullet}\right)$ is different for each coupling approach, and is defined the same way as in pressure MSFV method (see Eqs. 21, 22, 23, and 24).

Note that the temperature basis function formulation is slightly different with pressure basis function. This is due to the fact that the well source term in the energy balance equation is defined through the enthalpy flow. Therefore, there is no explicit relation connecting the well and the matrix or fracture temperature. As such, the well basis function is omitted in Eq. 29.

The temperature basis functions depend only on thermal conductivity. And since the thermal conductivity is considered to be constant in this work, the basis functions therefore do not need to be updated frequently as they will also remain constant. In models where the thermal conductivity is considered to be non-constant, then an adaptive update of the temperature basis functions is necessary (i.e., if $\lambda_{c}$ changes above a prescribed tolerance). As will be seen in the result 
section, this formulation is shown to be working well to interpolate the coarse-scale temperature values to the fine scale. Note that these thermal basis functions along with the flow basis functions form the full prolongation (interpolation) operator to map between coarse and fine scale values for flow and heat.

\subsection{Multiscale algebraic description and algorithm}

In the algebraic formulation of the MSFV method, i.e., AMS [36, 37], the multiscale procedure can be described by the prolongation $\mathcal{P}$ and the restriction $\mathcal{R}$ operators. The prolongation operator is a matrix constructed by the basis function values (interpolators) to map the coarse scale to fine scale solution. The restriction operator, on the other hand, is useful to map from fine scale to coarse scale. In finite-volume formulation, it acts as an integrator of all the fine scale fluxes, source/sink terms, as well as accumulation inside a primal coarse cell [36]. In this section, the algebraic description is explained in a generic way for both pressure and temperature calculation. More specifically, the prolongation operator reads

$\mathcal{P}=\left[\begin{array}{l}\mathcal{P}^{m} \\ \mathcal{P}^{f} \\ \mathcal{P}^{w}\end{array}\right]=\left[\begin{array}{lll}\mathcal{P}^{m m} & \mathcal{P}^{m f} & \mathcal{P}^{m w} \\ \mathcal{P}^{f m} & \mathcal{P}^{f f} & \mathcal{P}^{f w} \\ \mathcal{P}^{w m} & \mathcal{P}^{w f} & \mathcal{P}^{w w}\end{array}\right]$

where $\mathcal{P}^{*}$ stores the basis functions defined on domain $*$, $\Phi_{c, d}^{* \bullet}$, i.e.,

$\mathcal{P}^{*}=\left[\begin{array}{ccc|ccc|ccc}\vdots & \ldots & \vdots & \vdots & \ldots & \vdots & \vdots & \ldots & \vdots \\ \Phi_{1}^{* m} & \ldots & \Phi_{N_{c m}}^{* m} & \Phi_{1}^{* f_{1}} & \ldots & \Phi_{N_{c f} N_{f}}^{* f_{N_{f}}} & \Phi_{1}^{* w} & \ldots & \Phi_{N_{w}}^{* w} \\ \vdots & \ldots & \vdots & \vdots & \ldots & \vdots & \vdots & \ldots & \vdots\end{array}\right]$

In the totally independent coupling strategy, the submatrices $\mathcal{P}^{m f}$ and $\mathcal{P}^{f m}$ are zero matrices, resulting in a sparser prolongation operator than in the semi-dependent coupling strategy, where only $\boldsymbol{\mathcal { P }}^{f m}$ is zero. Note also that $\mathcal{P}^{* w}$ for temperature calculation has the column size of $N_{w, i n j}$, and $\mathcal{P}^{w *}$ has the row size of $N_{w, i n j}$ instead of $N_{w}$.

The MSFV restriction operator is defined as

$\boldsymbol{R}_{i, j}^{F V}=\left\{\begin{array}{lc}1, & \text { if fine cell } j \text { is in primal coarse cell } i \\ 0, & \text { otherwise }\end{array}\right.$,

and in MSFE method, the restriction is defined as the transpose of the prolongation operator, $\mathcal{R}^{F E}=\mathcal{P}^{T}$.

Now that both operators are defined, the coarse scale system in equation is written algebraically as

$\left(\boldsymbol{R} \boldsymbol{A}^{\nu} \mathcal{P}\right) \breve{\boldsymbol{x}}_{c}^{\nu+1 / 2}=\boldsymbol{R} \boldsymbol{f}^{\nu}$,

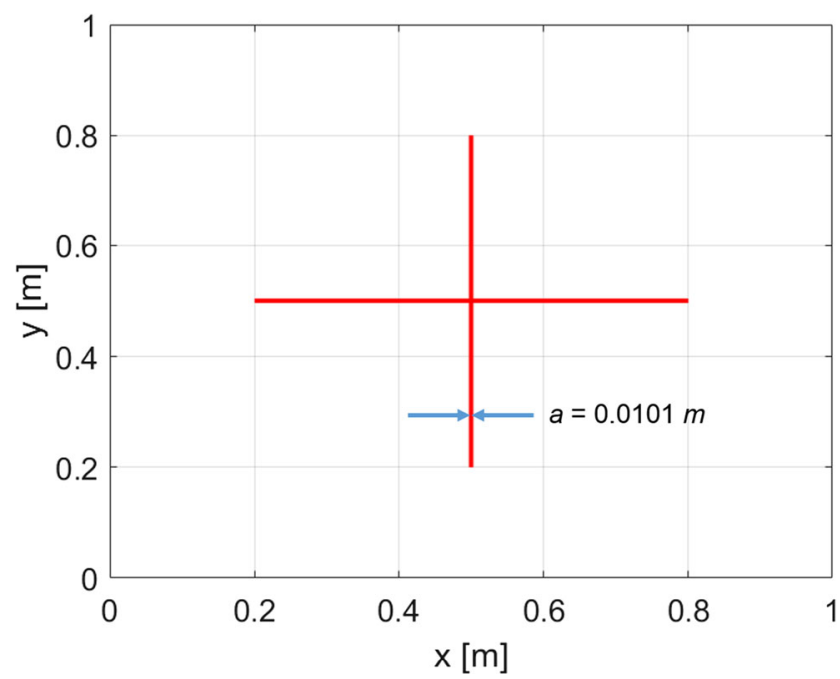

Fig. 4 Geometry of the "+"-shaped fracture networks crossing the reservoir for test case 1

where $\breve{\boldsymbol{x}}_{c}^{\nu+1 / 2}$ is the coarse scale solution (i.e., pressure or temperature), and the superscript $v+1 / 2$, indicating that this stage will be complemented by a second stage smoother to be explained later.

Note that in Eq. 33, $\left(\boldsymbol{R} \boldsymbol{A}^{\nu} \mathcal{P}\right)$ constructs the coarse system matrix $\boldsymbol{A}_{c}^{v}$. The approximate fine scale solution is found as

$\boldsymbol{x}^{\prime \nu+1 / 2}=\mathcal{P} \breve{\boldsymbol{x}}_{c}^{\nu+1 / 2}=\mathcal{P}\left(\mathcal{R} \boldsymbol{A}^{\nu} \mathcal{P}\right)^{-1} \mathcal{R} \boldsymbol{f}^{\nu}$,

or in residual form,

$\boldsymbol{\delta} \boldsymbol{x}^{\prime \nu+1 / 2}=\boldsymbol{P} \boldsymbol{\delta} \breve{\boldsymbol{x}}_{c}^{\nu+1 / 2}=\mathcal{P}\left(\boldsymbol{R} \boldsymbol{A}^{\nu} \mathcal{P}\right)^{-1} \boldsymbol{R} \boldsymbol{r}^{\nu}$,

where $\boldsymbol{r}^{v}$ is the fine-scale residual and is calculated as $\boldsymbol{r}^{v}=$ $\boldsymbol{f}^{v}-\boldsymbol{A}^{v} \boldsymbol{x}^{\prime \nu}$.

In each solver, both $\delta \boldsymbol{p}^{\nu+1 / 2}$ and $\delta \boldsymbol{T}^{\nu+1 / 2}$ are calculated first using multiscale operators (see Eq. 35), and then a $2^{\text {nd }}$ stage smoother (in this study, ILU(0) with $\boldsymbol{M}_{\boldsymbol{I L U}}{ }^{-1}$ operator [38]) is employed, i.e.,

$\delta \boldsymbol{x}^{\prime \nu+2 / 2}=\boldsymbol{M}_{\boldsymbol{I L U}}{ }^{-1} \boldsymbol{r}^{\nu}$.

Table 1 Simulation parameters for test case 1

\begin{tabular}{llll}
\hline Parameters & Values & Parameters & Values \\
\hline$L_{x}[\mathrm{~m}]$ & 1 & $p_{i}[\mathrm{~Pa}]$ & $1 \times 10^{7}$ \\
$L_{y}[\mathrm{~m}]$ & 1 & $p_{L}[\mathrm{~Pa}]$ & $2 \times 10^{7}$ \\
$\Delta x[\mathrm{~m}]$ & 0.01 & $p_{R}[\mathrm{~Pa}]$ & $1 \times 10^{7}$ \\
$\Delta y[\mathrm{~m}]$ & 0.01 & $C_{p r}[\mathrm{~J} / \mathrm{kg}-\mathrm{K}]$ & 920 \\
$t[\mathrm{~s}]$ & 20000 & $\rho_{r}\left[\mathrm{~kg} / \mathrm{m}^{3}\right]$ & 2650 \\
$\Delta t[\mathrm{~s}]$ & 200 & $\lambda_{c r}[\mathrm{~W} / \mathrm{m}-\mathrm{K}]$ & 1.5 \\
$\phi[-]$ & 0.1 & $T_{i}[\mathrm{~K}]$ & 500 \\
$k\left[\mathrm{~m}^{2}\right]$ & $10^{-15}$ & $T_{L}[\mathrm{~K}]$ & 300 \\
$a[\mathrm{~m}]$ & 0.0101 & $\varepsilon_{s}[-]$ & $10^{-2}$ \\
$k^{f}\left[\mathrm{~m}^{2}\right]$ & $8.50 \times 10^{-6}$ & & \\
\hline
\end{tabular}




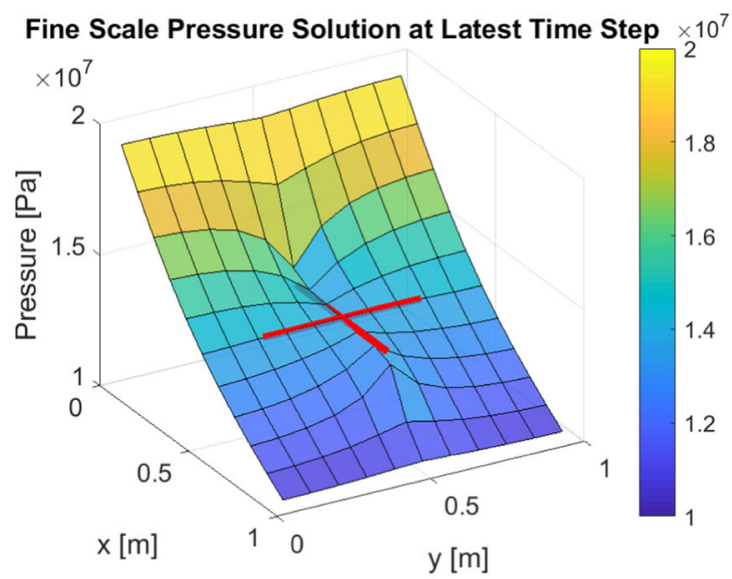

(a) EDFM pressure solution.

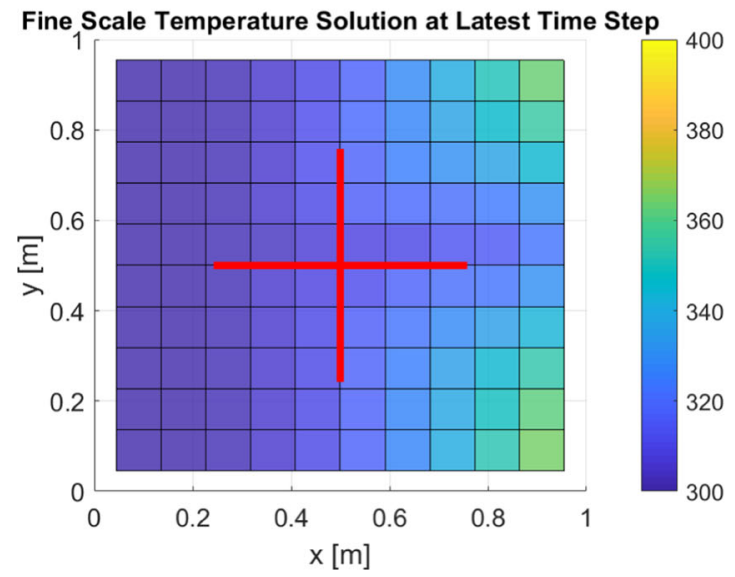

(c) EDFM temperature solution.

Fig. 5 EDFM pressure surface plot (a) and top view (b), and DNS pressure surface plot (c) and top view (d) at $t=20000 \mathrm{~s}$ for a reservoir model with the dimension of $1 m \times 1 \mathrm{~m}$. The EDFM model is resolved

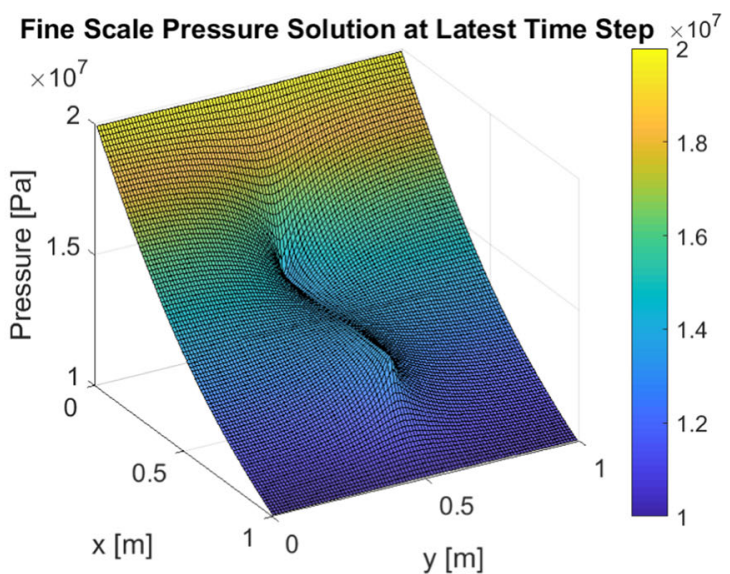

(b) DNS pressure solution.

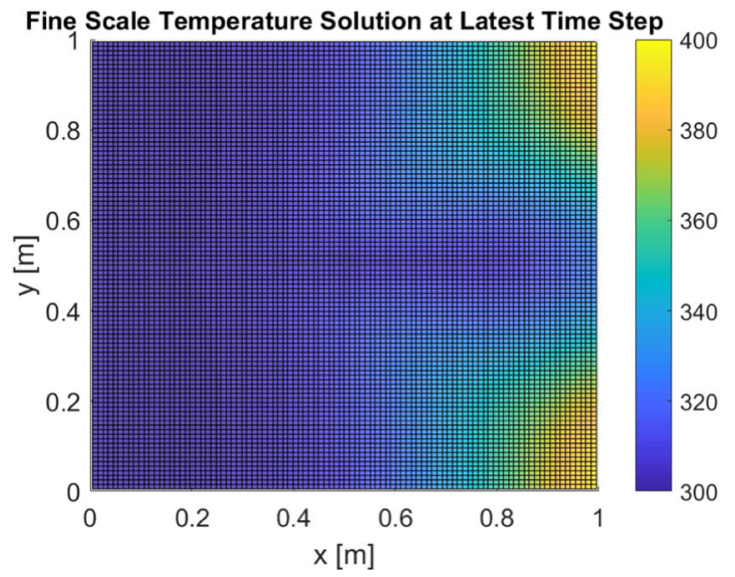

(d) DNS temperature solution.

with the resolution of $11 \times 11$ matrix cells and 14 fracture elements, while the DNS model is resolved with the resolution of $99 \times 99$ matrix cells
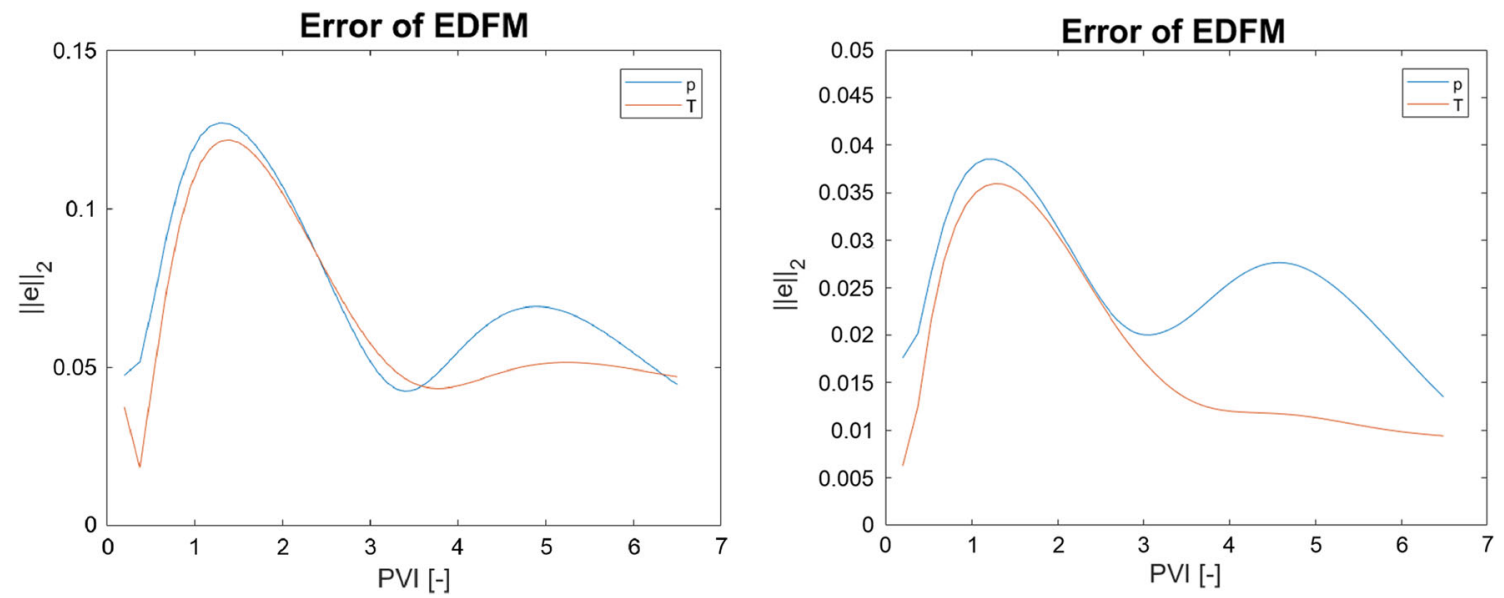

Fig. 6 Pressure and temperature errors of EDFM with respect to the DNS solutions used as reference. Different grid resolutions are used: $11 \times 11$ (left) and $33 \times 33$ (right) 
Here, we employ 5 ILU(0) iterations per stage. This twostage multiscale procedure is repeated iteratively until the norm of residual goes below the prescribed tolerance.

The approximate fine scale solution is finally calculated as $\boldsymbol{x}^{\prime \nu+1}=\boldsymbol{x}^{\prime \nu}+\boldsymbol{\delta} \boldsymbol{x}^{\prime \nu+1 / 2}+\boldsymbol{\delta} \boldsymbol{x}^{\prime \nu+2 / 2}$, where $\boldsymbol{x}^{\prime} \in$ $\left\{\boldsymbol{p}^{\prime}, \boldsymbol{T}^{\prime}\right\}$. The MS algorithms for pressure and temperature are presented in Algorithm 2.

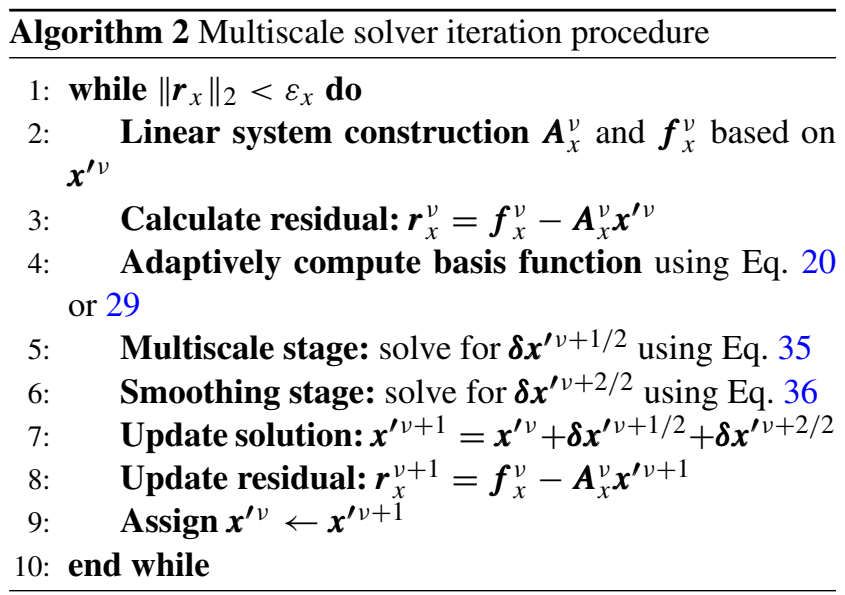

\section{Numerical results}

In this chapter, numerical results are presented first to validate the EDFM model for coupled flow-heat equations, and then to investigate the performance of the multiscale simulation strategy for fractured reservoirs.

\subsection{Test case 1: validation of EDFM}

In this test case, the fine scale EDFM model is validated by comparing it to the result of the fully resolved Direct Numerical Simulation (DNS), used as a reference. The DNS result is obtained by using a very fine grid such that the fractures are captured as equi-dimensional (heterogeneous) objects [15]. EDFM, on the other hand, imposes much coarser grids and models the impact of the explicit lower-dimensional fractures by introducing fracture-matrix connectivities.

The fracture aperture is $0.0101 \mathrm{~m}$, which can be fully resolved by imposing $99 \times 99$ DNS grid cells Fig. 4 . This aperture leads to the fracture permeability of $k^{f}=8.50 \times$ $10^{-6} \mathrm{~m}^{2}$. The simulation parameters are shown in Table 1 .

Figure 5 presents the pressure and temperature solutions obtained from EDFM and DNS simulators. Note that the EDFM solutions are obtained by imposing only $11 \times 11$ matrix cells and 14 fracture elements. It is clear that the EDFM solutions are in good agreement with the DNS reference ones.
Table 2 Simulation parameters for test case 2

\begin{tabular}{llll}
\hline Parameters & Values & Parameters & Values \\
\hline$L_{x}[\mathrm{~m}]$ & 99 & $p_{i}[\mathrm{~Pa}]$ & $1 \times 10^{7}$ \\
$L_{y}[\mathrm{~m}]$ & 99 & $p_{\text {inj }}[\mathrm{Pa}]$ & $2 \times 10^{7}$ \\
$\Delta x[\mathrm{~m}]$ & 1 & $p_{\text {prod }}[\mathrm{Pa}]$ & $1 \times 10^{7}$ \\
$\Delta y[\mathrm{~m}]$ & 1 & $C_{p r}[\mathrm{~J} / \mathrm{kg}-\mathrm{K}]$ & 840 \\
Coarsening & & & \\
$r a t i o[-]$ & 11 & $\rho_{r}\left[\mathrm{~kg} / \mathrm{m}^{3}\right]$ & 2700 \\
$t[$ years $]$ & 1 & $\lambda_{c r}[\mathrm{~W} / \mathrm{m}-\mathrm{K}]$ & 2.9 \\
$\phi[-]$ & 0.15 & $T_{i}[\mathrm{~K}]$ & 500 \\
$k\left[\mathrm{~m}^{2}\right]$ & $10^{-14}$ & $T_{i n j}[\mathrm{~K}]$ & 300 \\
$a[\mathrm{~m}]$ & 0.001 & $\varepsilon_{s}[-]$ & $10^{-2}$ \\
$k^{f}\left[\mathrm{~m}^{2}\right]$ & $8.33 \times 10^{-8}$ & $\varepsilon_{p}[-]$ & $10^{-6}$ \\
$P I[\mathrm{~m}]$ & 10 & $\varepsilon_{T}[-]$ & $10^{-1}$ \\
\hline
\end{tabular}

The following measures are considered for the error of pressure and temperature:

$\left\|e_{x}\right\|_{2}=\frac{\left\|\boldsymbol{x}_{\boldsymbol{E} \boldsymbol{D} \boldsymbol{F M}}-\boldsymbol{x}_{\boldsymbol{D N S}}\right\|_{2}}{\left\|\boldsymbol{x}_{\boldsymbol{D N S}}\right\|_{2}}$,

assuming $\left\|\boldsymbol{x}_{\boldsymbol{D N} \boldsymbol{S}}\right\|_{2} \neq 0$, where $\boldsymbol{x}$ is flow rate $\boldsymbol{q}$ for pressure and enthalpy flux $\boldsymbol{q}_{H}$ for temperature at both left and right boundary faces. The error norms for both pressure and temperature at different time steps are plotted and shown in Fig. 6. This figure also presents the EDFM error study at different times for the case when $33 \times 33$ EDFM grids are imposed, with 40 fracture elements. The EDFM errors are plotted against Pore Volume Injected (PVI) which is a non-dimensional time measure. The pressure errors are shown to be increasing slightly twice, and this is most likely because there are two pressure transient stages. Initially, the reservoir is hot and therefore water viscosity is lower and water density is higher, making the pressure gradient low. However, the injected water is cold and therefore increasing the pressure gradient in areas close to injection wells (which in turn decreasing the pressure farther from

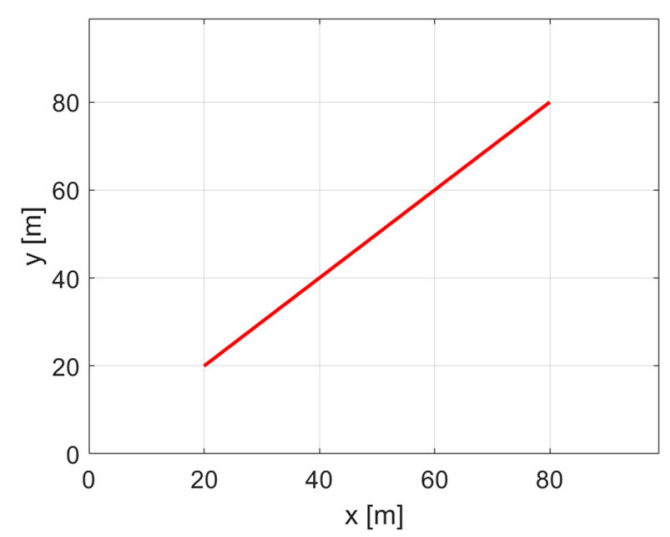

Fig. 7 Geometry of a single diagonal fracture crossing the reservoir for test case 2 


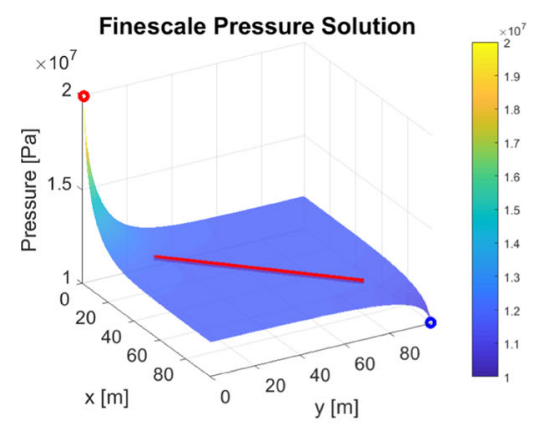

(a)

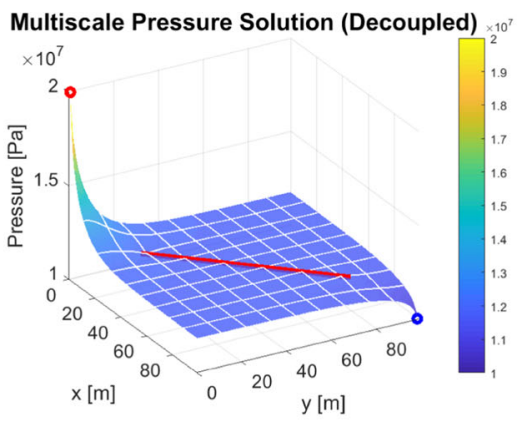

(b)

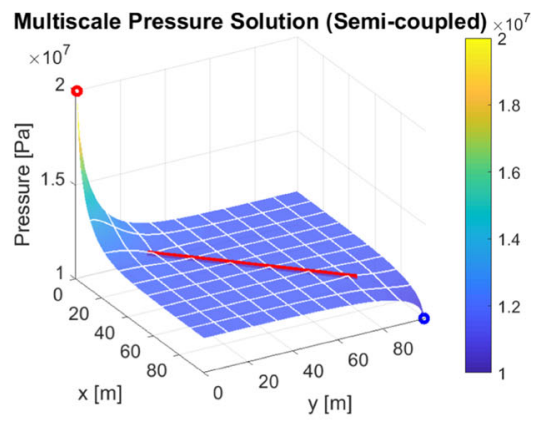

(c)

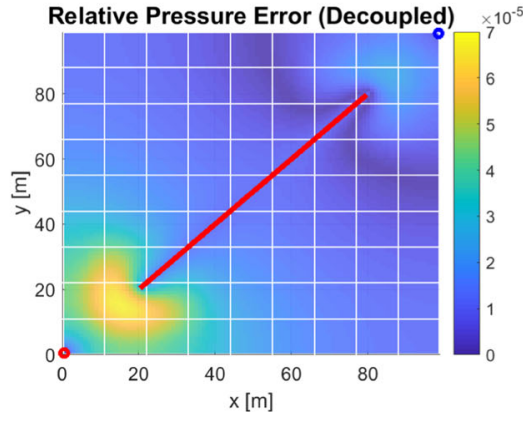

(d)

Fig. 8 Fine-scale reference pressure with $99 \times 99$ matrix and 85 fracture elements (a) and multiscale approximate pressure solutions obtained using independent (b) and semi-dependent coupling (c) methods with $9 \times 9$ coarse matrix and 8 fracture grid cell at convergence. The

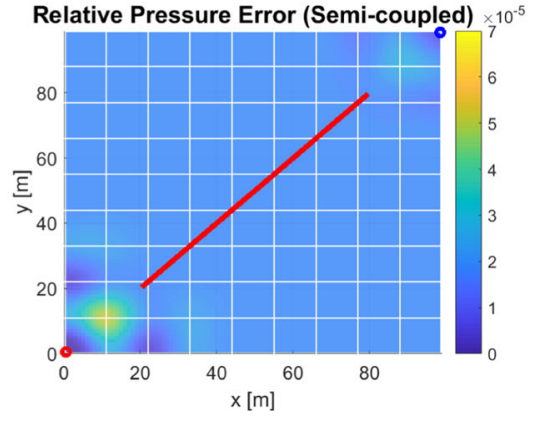

(e)

corresponding relative error norms ( $\mathrm{d}$ and e) are $\left\|e_{p}\right\|_{2}=2.65 \times 10^{-5}$ (independent coupling) and $\left\|e_{p}\right\|_{2}=2.18 \times 10^{-5}$ (semi-dependent coupling)

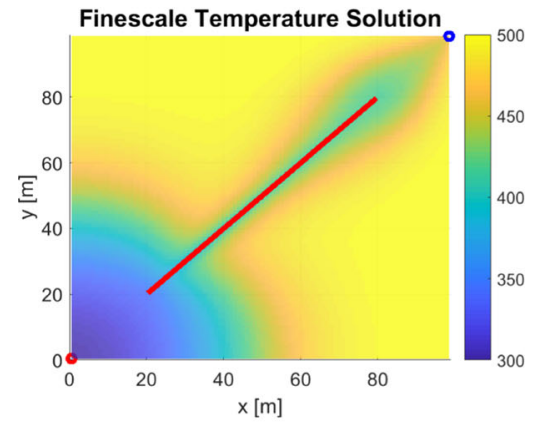

(a)

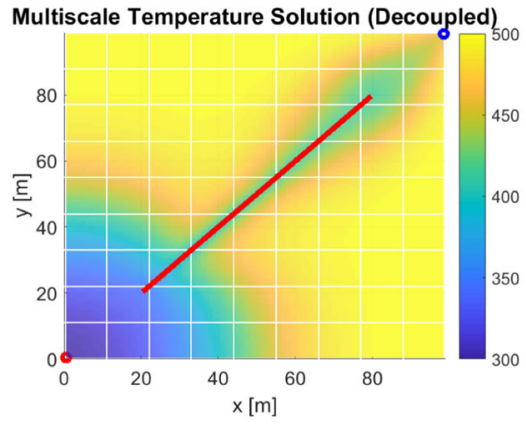

(b)

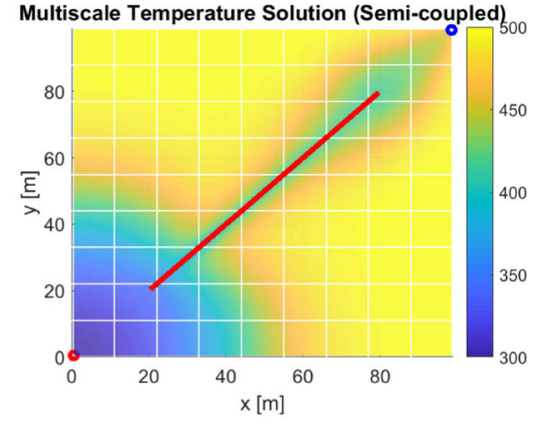

(c)

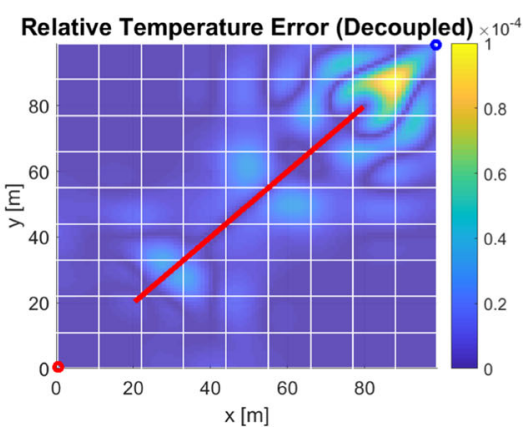

(d)

Fig. 9 Fine-scale reference temperature with $99 \times 99$ matrix and 85 fracture elements (a) and multiscale approximate temperature solutions obtained using independent (b) and semi-dependent coupling (c) methods with $9 \times 9$ coarse matrix and 8 fracture grid cells at

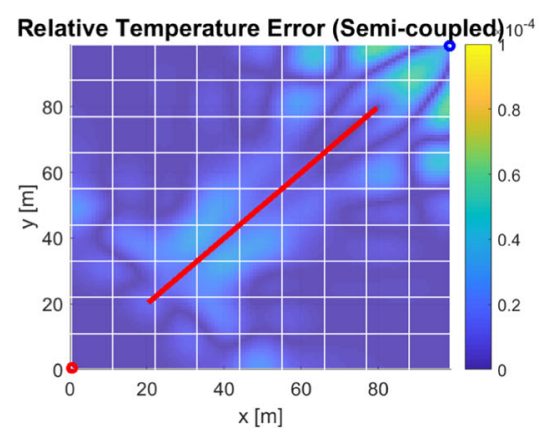

(e)

convergence. The corresponding relative error norms ( $\mathrm{d}$ and e) are $\left\|e_{T}\right\|_{2}=1.62 \times 10^{-5}$ (independent coupling) and $\left\|e_{T}\right\|_{2}=1.58 \times$ $10^{-5}$ (semi-dependent coupling) 


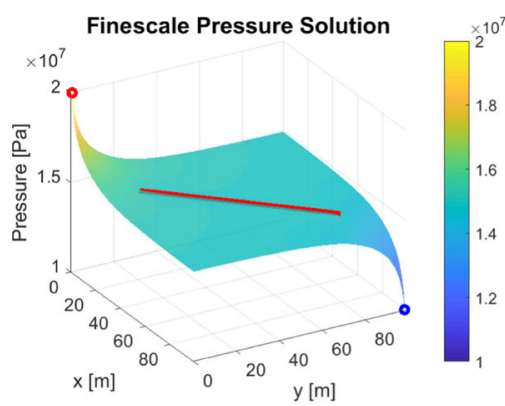

(a)

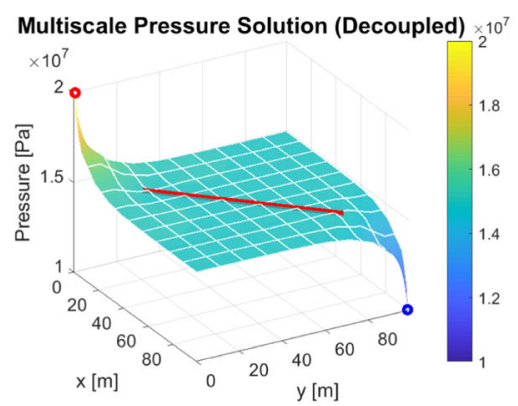

(b)

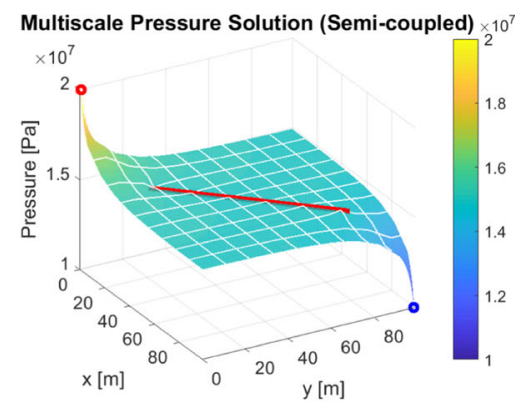

(c)

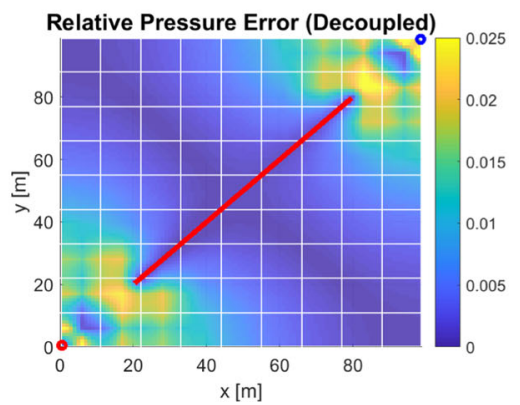

(d)

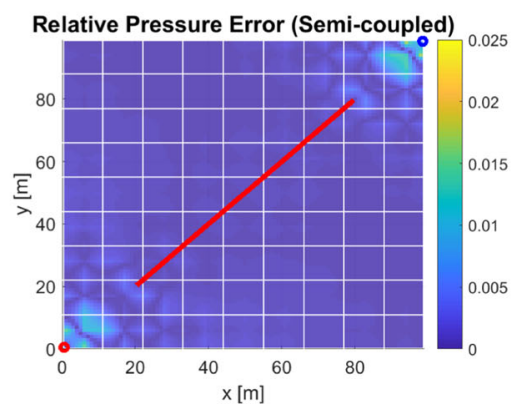

(e)

Fig. 10 Fine-scale reference pressure with $99 \times 99$ matrix and 85 fracture elements (a) and multiscale approximate pressure solutions obtained using independent (b) and semi-dependent coupling (c) methods with $9 \times 9$ coarse matrix and 8 fracture grid cells at the first iteration stage before smoothing. The corresponding relative error

norms (d and e) are $\left\|e_{p}\right\|_{2}=0.0081$ (independent coupling) and $\left\|e_{p}\right\|_{2}=0.0016$ (semi-dependent coupling). After 1 stage of smoothing these errors reduce to $\left\|e_{p}\right\|_{2}=0.0076$ (independent coupling) and $\left\|e_{p}\right\|_{2}=0.0008$ (semi-dependent coupling)

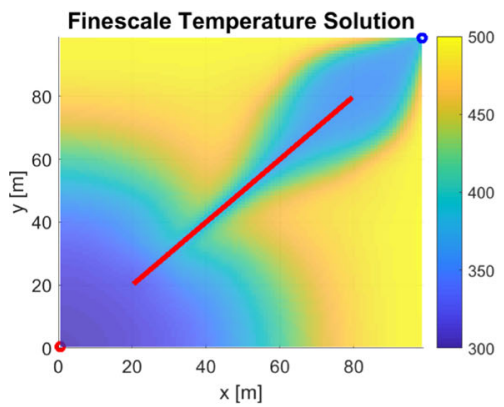

(a)

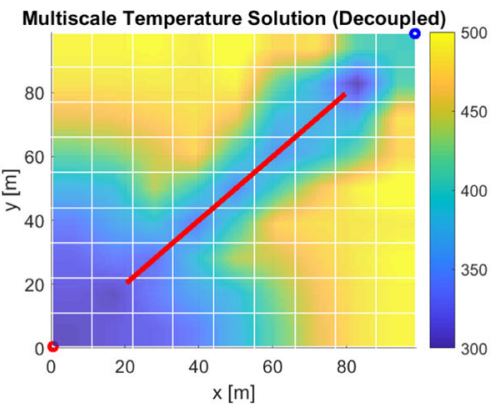

(b)

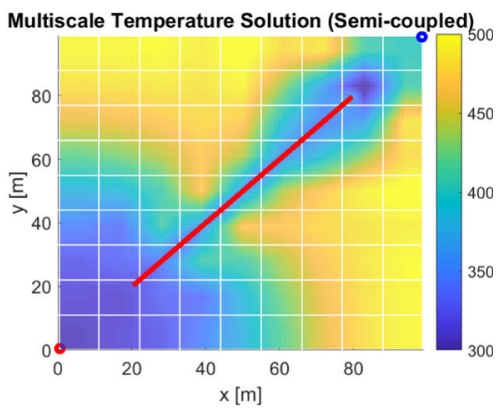

(c)

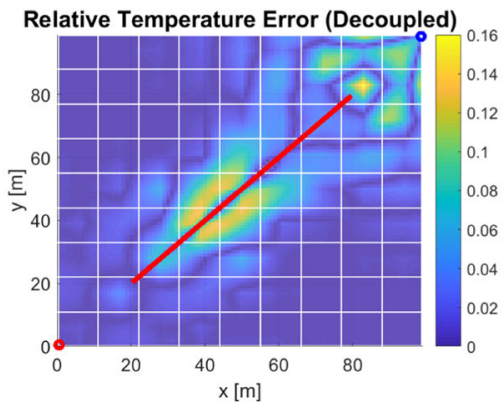

(d)

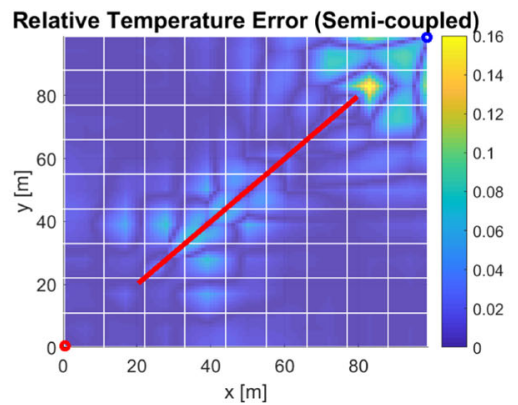

(e)

Fig. 11 Fine-scale reference temperature with $99 \times 99$ matrix and 85 fracture elements (a) and multiscale approximate temperature solutions obtained using independent (b) and semi-dependent coupling (c) methods with $9 \times 9$ coarse matrix and 8 fracture grid cells at the first iteration stage before smoothing. The corresponding relative error

norms (d and e) are $\left\|e_{T}\right\|_{2}=0.0343$ (independent coupling) and $\left\|e_{T}\right\|_{2}=0.0198$ (semi-dependent coupling). After 1 stage of smoothing these errors reduce to $\left\|e_{T}\right\|_{2}=0.0146$ (independent coupling) and $\left\|e_{T}\right\|_{2}=0.0035$ (semi-dependent coupling) 
Table 3 Simulation results of test case 2

\begin{tabular}{|c|c|c|c|c|}
\hline & \multicolumn{2}{|c|}{ Independent coupling } & \multicolumn{2}{|c|}{ Semi-dependent coupling } \\
\hline & $\left\|e_{p}\right\|_{2}$ & $\left\|e_{T}\right\|_{2}$ & $\left\|e_{p}\right\|_{2}$ & $\left\|e_{T}\right\|_{2}$ \\
\hline First iteration, no smoothing & 0.0081 & 0.0343 & 0.0016 & 0.0198 \\
\hline Converged & $2.65 \times 10^{-5}$ & $1.62 \times 10^{-5}$ & $2.18 \times 10^{-5}$ & $1.58 \times 10^{-5}$ \\
\hline
\end{tabular}
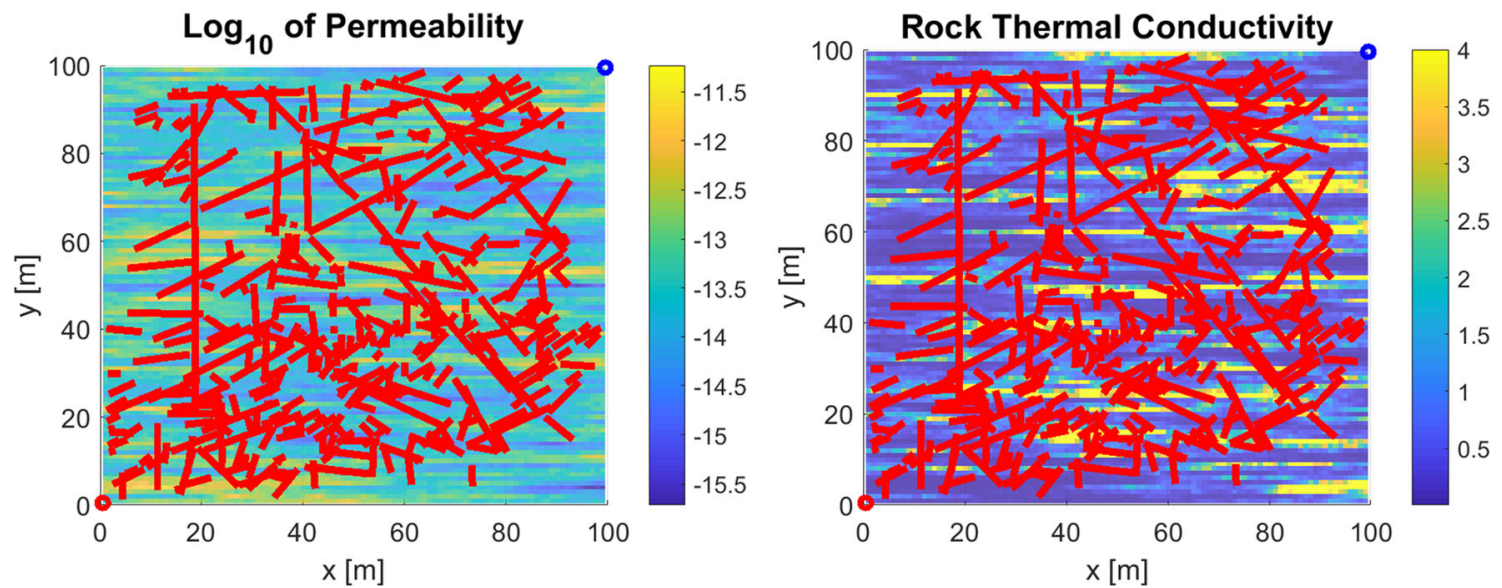

Fig. $12 \log _{10}$ of permeability (left) and average thermal conductivity (right) for reservoir model with the dimension of $100 \mathrm{~m} \times 100 \mathrm{~m}$, with the fine scale resolution of $100 \times 100$ cells and a coarsening ratio of 10

Table 4 Simulation parameters for test case 3

\begin{tabular}{llll}
\hline Parameters & Values & Parameters & Values \\
\hline$L_{x}[\mathrm{~m}]$ & 100 & $p_{i}[\mathrm{~Pa}]$ & $1 \times 10^{7}$ \\
$L_{y}[\mathrm{~m}]$ & 100 & $p_{\text {inj }}[\mathrm{Pa}]$ & $2 \times 10^{7}$ \\
$\Delta x[\mathrm{~m}]$ & 1 & $p_{\text {prod }}[\mathrm{Pa}]$ & $1 \times 10^{7}$ \\
$\Delta y[\mathrm{~m}]$ & 1 & $C_{p r}[\mathrm{~J} / \mathrm{kg}-\mathrm{K}]$ & 827 \\
Coarsening & & & \\
ratio $[-]$ & 10 & $\rho_{r}\left[\mathrm{~kg} / \mathrm{m}^{3}\right]$ & 2600 \\
$t[$ years $]$ & 0.25 & $T_{i}[\mathrm{~K}]$ & 500 \\
$\phi[-]$ & 0.1 & $T_{i n j}[\mathrm{~K}]$ & 300 \\
$a[\mathrm{~m}]$ & 0.001 & $\varepsilon_{s}[-]$ & $10^{-2}$ \\
$k^{f}\left[\mathrm{~m}^{2}\right]$ & $8.33 \times 10^{-8}$ & $\varepsilon_{p}[-]$ & $10^{-6}$ \\
$P I[\mathrm{~m}]$ & 10 & $\varepsilon_{T}[-]$ & $10^{-1}$ \\
\hline
\end{tabular}

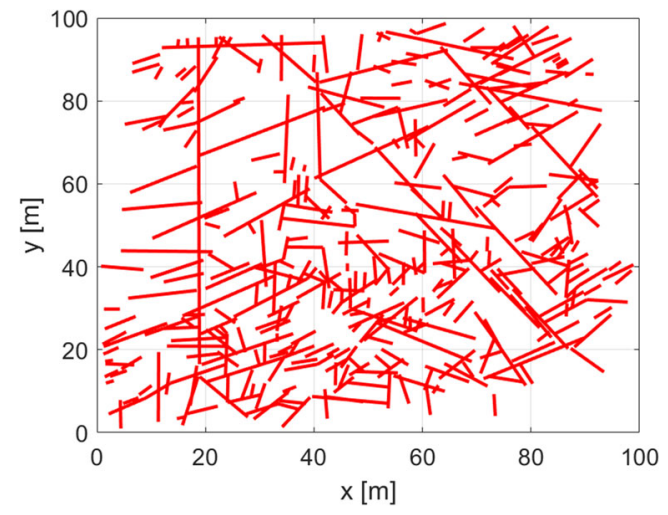

Fig. 13 Fracture geometry taken from outcrop data [39] 


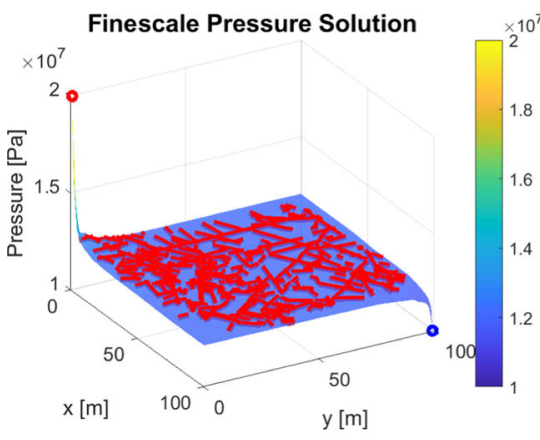

(a)

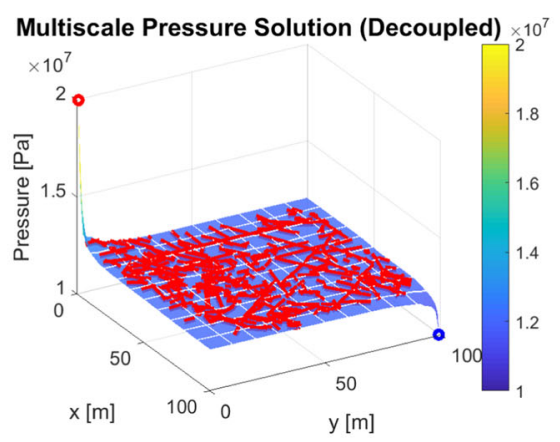

(b)

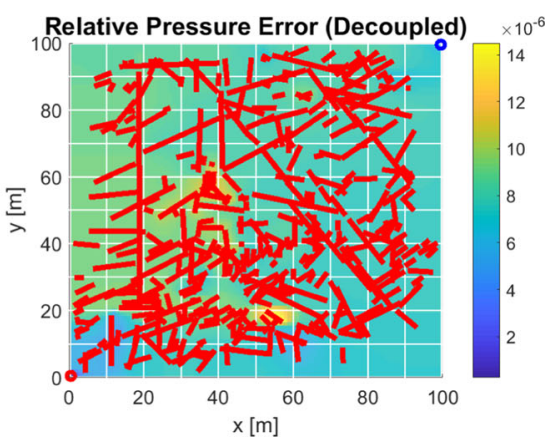

(c)
Fig. 14 Fine-scale reference pressure with $100 \times 100$ matrix and 3860 fracture elements (a) and multiscale approximate pressure solutions obtained using independent coupling $(\mathbf{b})$ method with $10 \times 10$ coarse

the injection wells). After the injection water gets farther into the reservoir, the pressure gradient close to the injection wells gets lower and the gradient farther in the reservoir gets higher until it reached semi-steady state. More specifically, the error is due to (1) significant difference between the grid resolutions imposed by each method and (2) the error of EDFM fracture model. Nevertheless, the two approaches are in good agreement.

\subsection{Test case 2: homogeneous reservoir with a diagonal fracture}

A quarter of a five-spot test case is considered in a homogeneous reservoir with a diagonal fracture. The simulation parameters are shown in Table 2. EDFM imposes 85 fracture and $99 \times 99$ matrix elements. The geometry of the fracture within the reservoir is shown in Fig. 7. The multiscale simulator imposes $9 \times 9$ coarse grids for matrix and 8 for fractures with two different coupling strategies for basis function calculation.

Figures 8 and 9 show the converged solution of both fine scale reference as well as multiscale pressure and matrix and 386 fracture grid cells at convergence. The corresponding relative error norm $(\mathbf{c})$ is $\left\|e_{p}\right\|_{2}=8.22 \times 10^{-6}$

temperature. The white lines shown in the plots are the primal coarse cell boundaries. The relative error norms of the multiscale solutions are $\left\|e_{p}\right\|_{2}=2.65 \times 10^{-5}$ and $\left\|e_{T}\right\|_{2}=1.62 \times 10^{-5}$ (independent coupling), and $\left\|e_{p}\right\|_{2}=2.18 \times 10^{-5}$ and $\left\|e_{T}\right\|_{2}=1.58 \times 10^{-5}$ (semidependent coupling). It is shown that both independent and semi-dependent coupling strategies result in very good results.

The multiscale pressure and temperature solutions at the first iteration (before smoothing) are also presented in Figs. 10 and 11, respectively, to show that the multiscale provides very good approximations even with no secondstage smoother nor any other (inner and outer) iterations. These results are also compared to the reference fine scale solutions, demonstrating the accuracy of the developed multiscale formulation.

Independent coupling for pressure basis function calculation results in slightly higher error at the fracture tips, where-as expected - the interaction of matrix and fracture domain is relatively high. Note that the temperature field experiences a rapid change in the location of the fracture, due to rapid transport of cold water through the fractures. As

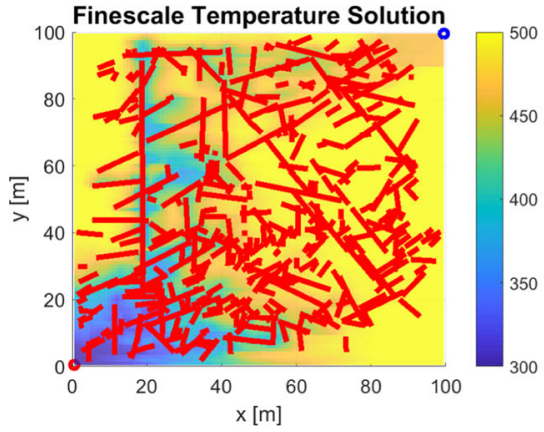

(a)

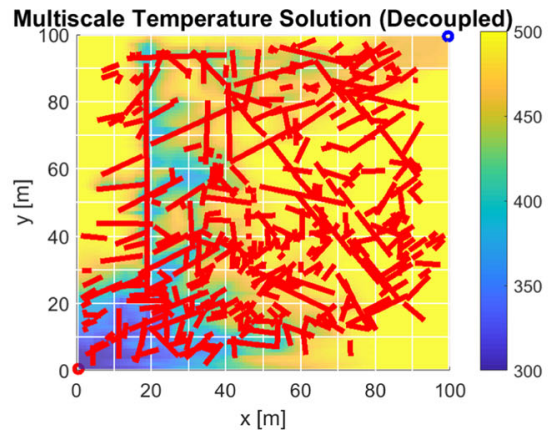

(b)

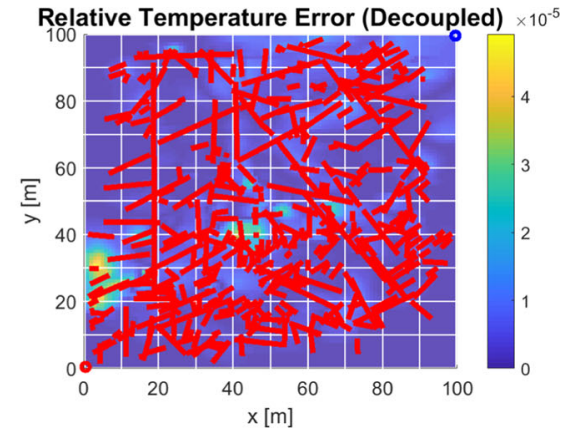

(c)

Fig. 15 Fine-scale reference temperature with $100 \times 100$ matrix and 3860 fracture elements (a) and multiscale approximate temperature solutions obtained using independent coupling (b) method with $10 \times 10$

matrix and 386 fracture grid cells at convergence. The corresponding relative error norm $(\mathbf{c})$ is $\left\|e_{T}\right\|_{2}=7.07 \times 10^{-6}$ 


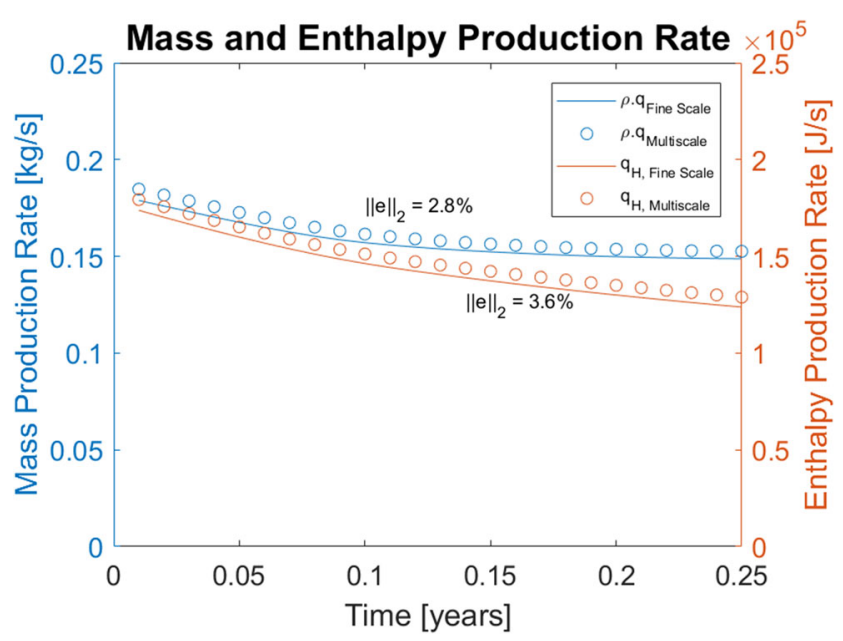

Fig. 16 Multiscale (dotted line) mass and enthalpy production rate compared with the fine scale (solid line) solutions for test case 3

such, the significant temperature contrast is created fairly quickly throughout the reservoir, leading to strong nonlinear time-dependent solution field. In the area near the midpoint of the fracture, the semi-dependent coupling provides a slightly lower error for temperature. This can be explained by the fact that in this area, pressure difference between matrix and fracture is lower and therefore, the heat exchange is more conduction dominated. Since the matrix-fracture coupling in the semi-dependent approach for temperature is formulated based on conduction, it leads to better approximation in this area. This is clear from results presented in Fig. 11. Nevertheless, as shown, the multiscale method can represent the complex solution field accurately, even with no smoothing stage.

At the first iteration stage, the relative error norms of the multiscale solution obtained before smoothing are $\left\|e_{p}\right\|_{2}=$ 0.0081 and $\left\|e_{T}\right\|_{2}=0.0343$ (independent coupling), and $\left\|e_{p}\right\|_{2}=0.0016$ and $\left\|e_{T}\right\|_{2}=0.0198$ (semi-dependent coupling). After 1 stage of smoothing, the errors are reduced to $\left\|e_{p}\right\|_{2}=0.0076$ and $\left\|e_{T}\right\|_{2}=0.0146$ (independent coupling) and $\left\|e_{p}\right\|_{2}=0.0008$ and $\left\|e_{T}\right\|_{2}=0.0035$ (semi-dependent coupling). The results are summarized in Table 3.

The semi-dependent coupling strategy leads to lower errors compared to the independent coupling, especially in the area surrounding the fracture. However, it does not bring much improvements. The errors obtained using independent coupling are not significant and could be resolved with several smoothing and iterations.

\subsection{Test case 3: fracture geometry from outcrop data}

A quarter of a five spot test case is considered in a heterogeneous reservoir with dense and complex fracture networks taken (by applied geologists of TU Delft) from outcrop data in Brazil [39]. The base-10 logarithm of permeability and average thermal conductivity are plotted in Fig. 12, and the simulation parameters are shown in Table 4. EDFM generates 3860 fracture and $100 \times 100$ matrix elements. The geometry of the fractures within the reservoir is shown in Fig. 13. The multiscale simulator imposes $10 \times 10$ coarse grids for matrix and 386 for fractures. For this test case, all the results presented are using independent coupling method.

As shown in test case 2, the decoupled approach approximates the solution really well. Therefore, for this test case, only the results obtained using the decoupled approach are shown for conciseness.

Figures 14 and 15 show the converged solution of both fine scale reference as well as multiscale pressure and temperature. The relative error norms of the multiscale solution obtained are $\left\|e_{p}\right\|_{2}=8.22 \times 10^{-6}$ and $\left\|e_{T}\right\|_{2}=$ $7.07 \times 10^{-6}$.

To make it more suitable for real field application and to further validate the multiscale method for this complex test case, mass and enthalpy production rate at the production

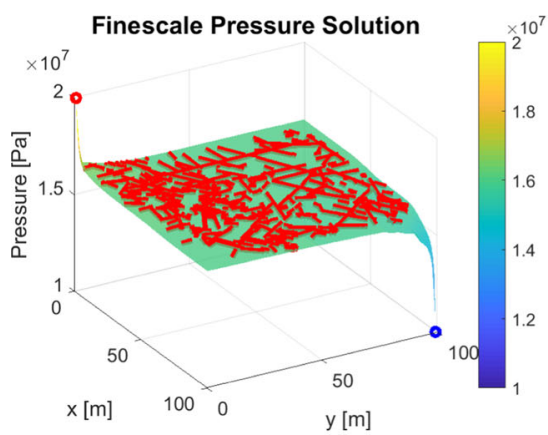

(a)

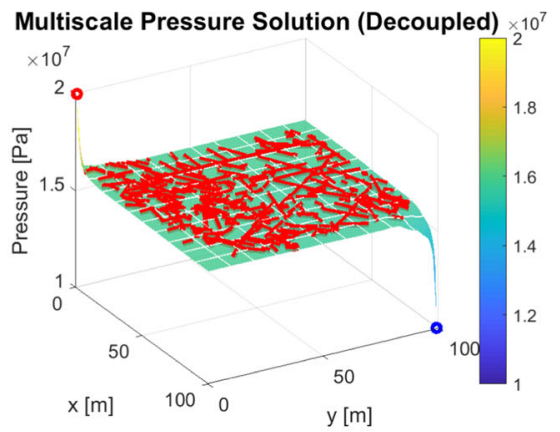

(b)

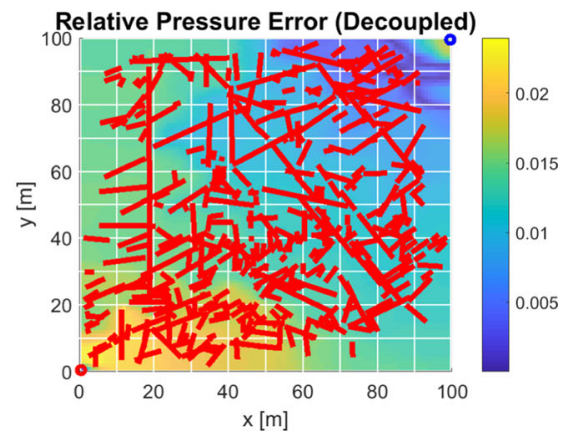

(c)
Fig. 17 Fine-scale reference pressure with $100 \times 100$ matrix and 3860 fracture elements (a) and multiscale approximate pressure solutions obtained using independent coupling (b) method with $10 \times 10$ coarse matrix and 386 fracture grid cells at the first iteration stage before smoothing. The corresponding relative error norm (c) is $\left\|e_{p}\right\|_{2}=$ 0.0111 . After 1 stage of smoothing this error reduces to $\left\|e_{p}\right\|_{2}=$ 0.0110 


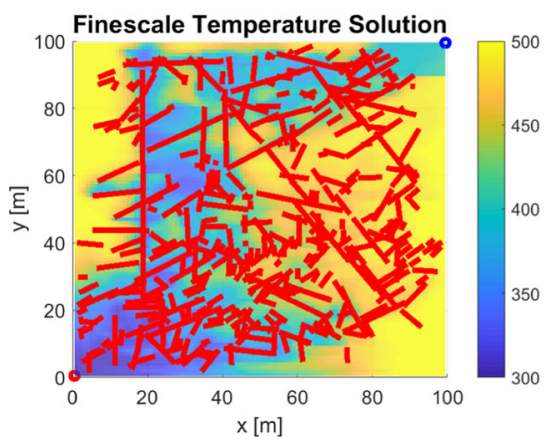

(a)

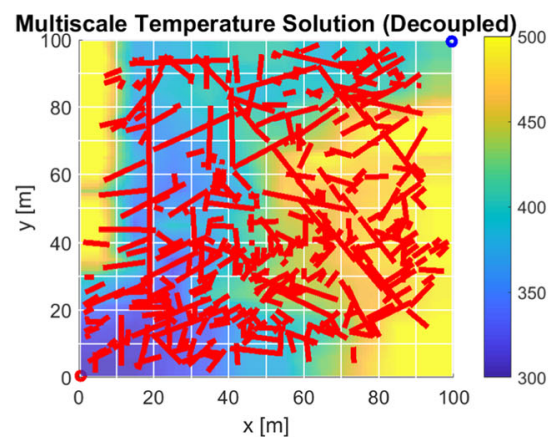

(b)

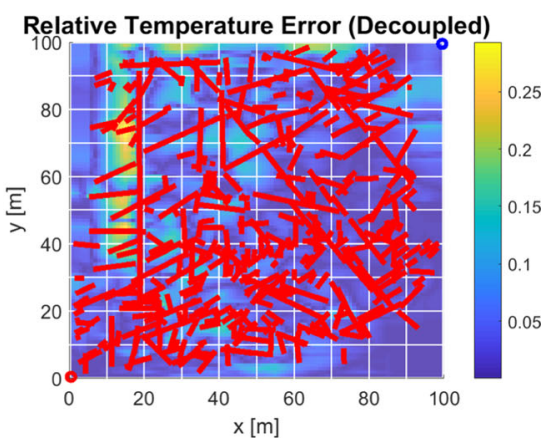

(c)
Fig. 18 Fine-scale reference temperature with $100 \times 100$ matrix and 3860 fracture elements (a) and multiscale approximate temperature solutions obtained using independent coupling (b) method with $10 \times 10$ matrix and 386 fracture grid cells at the first iteration stage before

well (marked by the blue dot on the top right hand side of the reservoir model) are calculated and compared with the values obtained using a direct solver, because they are important parameter outputs of a reservoir simulator that are useful for decision making. The result is shown in Fig. 16. In the plot, it is shown that both multiscale and the fine scale mass and enthalpy production rate have a very good match, with $\left\|e_{p}\right\|_{2}=0.028$ and $\left\|e_{T}\right\|_{2}=0.036$. The error of the enthalpy production rate is slightly higher due to the fact that the enthalpy production rate is calculated based on the mass production rate, and therefore the error from the mass production rate calculation is propagated. Nevertheless, the error is still relatively low and therefore acceptable.

The multiscale solutions for both pressure and temperature at the first iteration stage before smoothing, along with the fine scale reference solutions for comparison, are shown in Figs. 17 and 18. The corresponding relative error norms before smoothing are $\left\|e_{p}\right\|_{2}=0.0111$ and $\left\|e_{T}\right\|_{2}=0.0467$, which are relatively low for a complex model. After smoothing, the errors are further reduced to $\left\|e_{p}\right\|_{2}=0.0110$ and $\left\|e_{T}\right\|_{2}=0.0180$. The results of this test case are summarized in Table 5. The error reduction in pressure after the smoothing stage is not significant, while error reduction in temperature is more drastic because of the higher complexity of the heat transfer equation and the simplicity of the basis function used to calculate it. It is also clear that the temperature distribution in Fig. 18 (without smoothing) already captures the preferential flow path of the fluid.

Table 5 Simulation results of test case 3

\begin{tabular}{lll}
\hline & \multicolumn{2}{l}{ Independent coupling } \\
\cline { 2 - 3 } & $\left\|e_{p}\right\|_{2}$ & $\left\|e_{T}\right\|_{2}$ \\
\hline First iteration, no smoothing & 0.0111 & 0.0467 \\
Converged & $8.22 \times 10^{-6}$ & $7.07 \times 10^{-6}$ \\
\hline
\end{tabular}

smoothing. The corresponding relative error norm (c) is $\left\|e_{T}\right\|_{2}=$ 0.0467. After 1 stage of smoothing this error reduces to $\left\|e_{T}\right\|_{2}=$ 0.0180

From the results obtained, it can be concluded that independent coupling method gives reasonably good approximations, even before the smoothing stage for a heterogeneous system and very dense fracture networks.

\section{Conclusion}

In this work, a multiscale method for coupled single-phase flow-heat equations in fractured reservoirs was developed. The method avoids excessive upscaling of the parameters, and honours fine-scale heterogeneity in construction of the coarse-scale system for both flow and heat equations. This is achieved by formulating flow and temperature basis functions, allowing for the accurate map between fine and coarse scale solutions. The coupling between the equations was treated by a sequential implicit framework, where both pressure and temperature systems were solved by a MSFV method. The multiscale formulation was enriched due to the presence of the fractures, with two coupling approaches for local basis functions of each solver. An EDFM approach was adapted to the framework, which allows for independent grids for matrix and fractures. This further facilitated the convenient multiscale formulation and implementation, as totally independent coarse grids were also imposed on matrix and fractures. Test cases were performed first to validate the implementation of the simulator (via comparing its results with a DNS approach), and then to systematically assess the performance of the multiscale method for heterogeneous and highly fractured media. A fracture formation from a real-field outcrop was also considered to illustrate the capacity of the algorithm in addressing complex fracture networks.

Although we employed the MSFV iterations to reach convergence in our sequential implicit framework, one can stop iterations before convergence is reached. Specially, as shown in the results, the initial multiscale approximate 
solutions in presence of heterogeneity and fractures were close to the fine-scale solution at the same stage of iteration. The tolerance to stop iterations of a conservative multiscale solver needs to be defined based on the influence of the solution in the overall accuracy of the coupled solutions, the stability of the time-dependent solutions, and the uncertainty within the parameters. Similar to previous studies for coupled flow and transport [40], such a study is needed for coupled P-T as a future work. Specially, in the presence of strong coupling one may consider formulating a multiscale methodology for fully implicit systems [41, 42].

As for the multiscale basis functions, to exploit the maximum efficiency, the temperature basis function was formulated based on the elliptic part of the energy conservation equation (i.e., the conduction term). Numerical results showed that such an approach is well suited for the considered single-phase fluid-dynamic system, i.e., it leads to accurate results even without smoothing stage.

In this work, a robust approach for solving the coupled pressure and temperature equations in fractured heterogeneous reservoirs was developed. The results presented show promising framework for further developments for fieldscale enhanced geothermal systems. Future developments need to consider multiphase (including steam) effects for fluid and the geo-mechanical effects (including fracture activations or closures and propagation) for solid rock.

Acknowledgments Hadi Hajibeygi acknowledges the grant from Ministerium für Wissenschaft, Forschung und Kunst Baden-Württemberg and SimTech Centre of Stuttgart University.

Open Access This article is distributed under the terms of the Creative Commons Attribution 4.0 International License (http:// creativecommons.org/licenses/by/4.0/), which permits unrestricted use, distribution, and reproduction in any medium, provided you give appropriate credit to the original author(s) and the source, provide a link to the Creative Commons license, and indicate if changes were made.

\section{Appendix A: Fluid model}

All water properties are calculated using curve fitting approach with the correlations provided by the International Association for the Properties of Water and Steam Industrial Formulation 1997 (IAPWS-If97) [43]. Following the literature [30], water density, internal energy and enthalpy read

$\rho_{w} \approx \rho_{w s}(T)\left[1+c_{w}(T)\left(p-p_{s}(T)\right)\right]$,

$u_{w} \approx u_{w s}+C_{p w}\left(T-T_{s}\right)$,

and

$h_{w}=u_{w}+\frac{p}{\rho_{w}}$.
Here, $\rho_{w s}$ and $u_{w s}$ are, respectively, water density and internal energy at saturation condition $\left(p_{s}\right.$ and $\left.T_{s}\right)$. In this work, after curve fitting, the saturation density function reads

$\rho_{w s}(T)=\left\{\begin{array}{c}-0.0032 T^{2}+1.7508 T+757.5 T \leq 623.15 K \\ -0.5214 T^{2}+652.73 T-203714 T>623.15 K\end{array}\right.$

The isothermal compressibility values are calculated as a function of $T$ as

$c_{w}(T)=\left(0.0839 T^{2}-64.593 T+12437\right) \times 10^{-12}$,

$$
\text { for } 273 K<T<647 K \text {. }
$$

The saturation pressure, $p_{s}$, is calculated based on IAPWS-If97 [43] as

$p_{s}=\left[\frac{2 C}{-B+\left(B^{2}-4 A C\right)^{0.5}}\right]^{4} \times 10^{6} \mathrm{~Pa}$,

with the validity range of $273.15 K \leq T \leq 647.096 \mathrm{~K}$ (critical point). Here,

$A=\vartheta^{2}+n_{1} \vartheta+n_{2}$,

$B=n_{3} \vartheta^{2}+n_{4} \vartheta+n_{5}$,

and

$C=n_{6} \vartheta^{2}+n_{7} \vartheta+n_{8}$

hold, with

$\vartheta=T+\frac{n_{9}}{T-n_{10}}$.

Moreover, the empirical parameters, $n_{i}$, are shown in Table 6.

The combination of $u_{w s}=420 \mathrm{~kJ} / \mathrm{kg}, C_{p w}=4.2 \mathrm{~kJ} / \mathrm{kg}$, and $T_{s}=373 \mathrm{~K}$ was found to provide the best fitting values for internal energy calculation. More precisely, compared with the data, the density relative error norms were below $1 \%$ in most regions and $2.2 \%$ near the critical point. Similarly, the internal energy errors were less than $6 \%$.

Table 6 Coefficients of correlation for saturation pressure and temperature calculation from [43]

\begin{tabular}{ll}
\hline$i$ & $n_{i}$ \\
\hline 1 & $0.11670521452767 \times 10^{4}$ \\
2 & $-0.72421316703206 \times 10^{6}$ \\
3 & $-0.17073846940092 \times 10^{2}$ \\
4 & $0.12020824702470 \times 10^{5}$ \\
5 & $-0.32325550322333 \times 10^{7}$ \\
6 & $0.14915108613530 \times 10^{2}$ \\
7 & $-0.48232657361591 \times 10^{4}$ \\
8 & $0.40511340542057 \times 10^{6}$ \\
9 & -0.23855557567849 \\
10 & $0.65017534844798 \times 10^{3}$
\end{tabular}


Therefore, the presented fitted function has reasonable accuracy at temperature range of $273.15 K \leq T \leq$ $647.096 \mathrm{~K}$ and $p_{s}(T) \leq p \leq 22.064 M P a$, which is the single phase liquid region. Finally, the water viscosity [44] reads

$\mu_{w}(T)=2.414 \times 10^{-5} \times 10^{\frac{247.8}{T-140}}$ Pa.s.

\section{Appendix B: Fracture coordinates for the test cases}

In this appendix, the fracture coordinates for test cases 12 are presented. For test case 3, readers are referred to [39] due to the high number of fractures in the model.

The fractures are defined by two points: A and B and the $x$ and $y$ coordinates are given in the tables.

\section{B.1 Fracture coordinates for test case 1}

The fracture coordinates for test case 1 are listed in Table 7.

Table 7 Fracture coordinates for test case 1

\begin{tabular}{lllll}
\hline$N_{f}$ & $x_{A}[\mathrm{~m}]$ & $y_{A}[\mathrm{~m}]$ & $x_{B}[\mathrm{~m}]$ & $y_{B}[\mathrm{~m}]$ \\
\hline 1 & 0.2 & 0.5 & 0.8 & 0.5 \\
2 & 0.5 & 0.2 & 0.5 & 0.8 \\
\hline
\end{tabular}

\section{B.2 Fracture coordinates for test case 2}

The fracture coordinates for test case 2 are listed in Table 8 .

Table 8 Fracture coordinates for test case 2

\begin{tabular}{lllll}
\hline$N_{f}$ & $x_{A}[\mathrm{~m}]$ & $y_{A}[\mathrm{~m}]$ & $x_{B}[\mathrm{~m}]$ & $y_{B}[\mathrm{~m}]$ \\
\hline 1 & 20 & 20 & 80 & 80 \\
\hline
\end{tabular}

\section{References}

1. Berkowitz, B.: Characterizing flow and transport in fractured geological media: a review. Adv. Water Resour. 25(8-12), 861884 (2002)

2. Sakhaee-Pour, A., Wheeler, M.F.: Effective flow properties for cells containing fractures of arbitrary geometry. SPE J. 21(3), 965-980 (2016)

3. Gläser, D., Helmig, R., Flemisch, B., Class, H.: A discrete fracture model for two-phase flow in fractured porous media. Adv. Water Resour. 110, 335-348 (2017)

4. Ahmed, R., Edwards, M.G., Lamine, S., Huisman, B.A.H., Pal, M.: Control-volume distributed multi-point flux approximation coupled with a lower-dimensional fracture model. J. Comput. Phys. 284, 462-489 (2015)

5. Flemisch, B., Berre, I., Boon, W., Fumagalli, A., Schwenck, N., Scotti, A., Stefansson, I., Tatomir, A.: Benchmarks for singlephase flow in fractured porous media. Adv. Water Resour. 111, 239-258 (2018)

6. Geiger-Boschung, S., Matthäi, S.K., Niessner, J., Helmig, R.: Black-oil simulations for three-component, three-phase flow in fractured porous media. SPE J. 14(2), 338-354 (2009)

7. Reichenberger, V., Jakobs, H., Bastian, P., Helmig, R.: A mixeddimensional finite volume method for two-phase flow in fractured porous media. Adv. Water Resour. 29(7), 1020-1036 (2006)

8. Dietrich, P., Helmig, R., Sauter, M., Hötzl, H., Köngeter, J., Teutsch, G.: Flow and Transport in Fractured Porous Media, 1st edn. Springer, Berlin (2005)

9. Singh, G., Pencheva, G., Kumar, K., Wick, T., Ganis, B., Wheeler, M.F.: Impact of accurate fractured reservoir flow modeling on recovery predictions. In: SPE Hydraulic Fracturing Technology Conference. The Woodlands, Texas (2014)

10. Sandve, T.H., Keilegavlen, E., Nordbotten, J.M.: Physics-based preconditioners for flow in fractured porous media. Water Resour. Res. 50, 1357-1373 (2014)

11. Fumagalli, A., Zonca, S., Formaggia, L.: Advances in computation of local problems for a flow-based upscaling in fractured reservoirs. Math. Comput. Simul. 137, 299-324 (2017)

12. Fumagalli, A., Pasquale, L., Zonca, S., Micheletti, S.: An upscaling procedure for fractured reservoirs with embedded grids. Water Resour. Res. 52(8), 6506-6525 (2016)

13. Li, L., Lee, S.H.: Efficient field-scale simulation of black oil in naturally fractured reservoir through discrete fracture networks and homogenized media. SPE Reserv. Eval. Eng. 11(4), 750-758 (2008)

14. Lee, S.H., Lough, M.F., Jensen, C.L.: Hierarchical modeling of flow in naturally fractured formations with multiple length scales. Water Resour. Res. 37(3), 443-455 (2001)

15. Hajibeygi, H., Karvounis, D., Jenny, P.: A hierarchical fracture model for the iterative multiscale finite volume method. J. Comput. Phys. 230(24), 8729-8743 (2011)

16. Moinfar, A., Narr, W., Hui, M.-H., Mallison, B., Lee, S.H.: Comparison of discrete-fracture and dual-permeability models for multiphase flow in naturally fractured reservoirs. In: SPE Reservoir Simulation Symposium. The Woodlands, Texas (2011)

17. Pluimers, S.: Hierarchical Fracture Modeling Approach. Master's thesis. Delft University of Technology (2015)

18. Moinfar, A., Varavei, A., Sepehrnoori, K., Johns, R.: Development of an efficient embedded discrete fracture model for 3D compositional reservoir simulation in fractured reservoirs. SPE J. 19, 289-303 (2014)

19. Norbeck, J.H., McClure, M.W., Lo, J.W., Horne, R.N.: An embedded fracture modeling framework for simulation of hydraulic fracturing and shear stimulation. Comput. Geosci. 20(1), 1-18 (2016)

20. Tene, M., Bosma, S.B.M., Al Kobaisi, M.S., Hajibeygi, H.: Projection-based embedded discrete fracture model (pEDFM). Adv. Water Resour. 105, 205-216 (2017)

21. Jenny, P., Lee, S.H., Tchelepi, H.A.: Multi-scale finite-volume method for elliptic problems in subsurface flow simulation. J. Comput. Phys. 187(1), 47-67 (2003)

22. Hajibeygi, H., Bonfigli, G., Hesse, M.A., Jenny, P.: Iterative multiscale finite-volume method. J. Comput. Phys. 227(19), 8604-8621 (2008)

23. Kozlova, A., Li, Z., Natvig, J.R., Watanabe, S., Zhou, Y., Bratvedt, K., Lee, S.H.: A real-field multiscale black-oil reservoir simulator. In: SPE Reservoir Simulation Symposium, Houston (2015) 
24. Tene, M., Al Kobaisi, M.S., Hajibeygi, H.: Algebraic multiscale method for flow in heterogeneous porous media with embedded discrete fractures (F-AMS). J. Comput. Phys. 321, 819-845 (2016)

25. Shah, S., Moyner, O., Tene, M., Lie, K., Hajibeygi, H.: The multiscale restriction smoothed basis method for fractures porous media (F-MsRSB). J. Comput. Phys. 318, 36-57 (2016)

26. Efendiev, Y., Lee, S.H., Li, G., Yao, J., Zhang, N.: Hierarchical multiscale modeling for flows in fractured media using generalized multiscale finite element method. GEM - Int. J. Geomath. 6(2), 141-162 (2015)

27. Natvig, J.R., Skaflestad, B., Bratvedt, F., Bratvedt, K., Lie, K.-A., Laptev, V., Khataniar, S.: Multiscale mimetic solvers for efficient streamline simulation of fractured reservoirs. SPE J. 16(4), 880888 (2011)

28. Karvounis, D.C., Jenny, P.: Adaptive hierarchical fracture model for enhanced geothermal systems. Multiscale Model. Simul. 14(1), 207-231 (2016)

29. Peaceman, D.W.: Interpretation of well-block pressures in numerical reservoir simulation. In: SPE-AIME Annual Fall Technical Conference and Exhibition. Denver (1977)

30. Coats, K.H.: Geothermal reservoir modelling. In: SPE Annual Fall Technical Conference and Exhibition. Denver (1977)

31. Lampe, V.: Modelling Fluid Flow and Heat Transport in Fractured Porous Media. Master's thesis. University of Bergen (2013)

32. Suárez-Arriaga, M.C.: Local thermal non-equilibrium interfacial interactions in heterogeneous reservoirs - divergence of numerical methods to simulate the fluid and heat flow. In: 40th Workshop on Geothermal Reservoir Engineering. Stanford University, Stanford (2016)

33. Wallis, J., Tchelepi, H.A.: Apparatus, method and system for improved reservoir simulation using an algebraic cascading class linear solver. US Patent 7,684,967 (2010)

34. Tene, M., Wang, Y., Hajibeygi, H.: Adaptive algebraic multiscale solver for compressible flow in heterogeneous porous media. J. Comput. Phys. 300, 679-694 (2015)
35. Hajibeygi, H., Jenny, P.: Multiscale finite-volume method for parabolic problems arising from compressible multiphase flow in porous media. J. Comput. Phys. 228(14), 5129-5147 (2009)

36. Wang, Y., Hajibeygi, H., Tchelepi, H.A.: Algebraic multiscale solver for flow in heterogeneous porous media. J. Comput. Phys. 259, 284-303 (2014)

37. Zhou, H., Tchelepi, H.A.: Two-stage algebraic multiscale linear solver for highly heterogeneous reservoir models. SPE J. 17(2), 523-539 (2012)

38. Saad, Y.: Iterative Methods for Sparse Linear Systems, 2nd edn. Society for Industrial and Applied Mathematics (2003)

39. Bisdom, K., Gauthier, B.D.M., Bertotti, G., Hardebol, N.J.: Calibrating discrete fracture-network models with a carbonate three-dimensional outcrop fracture network: implications for naturally fractured reservoir modeling. AAPG Bull. 98(7), 13511376 (2014)

40. Hajibeygi, H., Lee, S.H., Lunati, I.: Accurate and efficient simulation of multiphase flow in a heterogeneous reservoir with error estimate and control in the multiscale finite-volume framework. SPE J. 17(4), 1071-1083 (2012)

41. Wong, Z.Y., Rin, R., Tchelepi, H., Horne, R.: Comparison of fully implicit and sequential implicit formulation for geothermal reservoir simulations. In: 42nd Workshop on Geothermal Reservoir Engineering. Stanford University, Stanford (2017)

42. Cusini, M., van Kruijsdijk, C., Hajibeygi, H.: Algebraic dynamic multilevel (adm) method for fully implicit simulations of multiphase flow in porous media. J. Comput. Phys. 314, 60-79 (2016)

43. Wagner, W., Kretzschmar, H.-J.: International Steam TablesProperties of Water and Steam Based on the Industrial Formulation IAPWS-IF97, 2nd edn. Springer, Berlin (2008)

44. Al-Shemmeri, T.T.: Engineering Fluid Mechanics, chap 1, p 18. Bookboon (2012) 